\title{
The RNA-binding protein Orb2 is associated with microcephaly and supports centrosome asymmetry in neural stem cells
}

\author{
Beverly V. Robinson ${ }^{1,2}$, Junnan Fang ${ }^{1,3}$, Dipen S. Mehta ${ }^{3,4}$, Joseph Buehler ${ }^{5}$, and Dorothy A. \\ Lerit $^{1 *}$
}

Author Affiliations:

1 Department of Cell Biology, Emory University School of Medicine, Atlanta, GA 30322

2 Genetics and Molecular Biology Graduate Program, Emory University, Atlanta, GA 30322

3 These authors contributed equally

4 College of Science and Mathematics, Augusta University, Augusta, GA 30912

5 Biochemistry, Cell, and Developmental Biology Graduate Program, Emory University, Atlanta, GA 30322

* Correspondence: dlerit@emory.edu

Running title: CPEB ortholog Orb2 modulates centrosome activity

Keywords: centrosome, asymmetry, neural stem cell, Orb2, CPEB, RNA-binding protein, posttranscriptional regulation, MTOC

Funding information: This work was supported by NIH grant T32GM008490 (BVR), American Heart Association postdoctoral fellowship 20POST35210023 (to JF) and NIH grant R01GM138544 (to DAL). 


\section{Abstract}

37 To maintain a balance of self-renewal versus neurogenesis, neural stem cells (NSCs) undergo

38 repeated cycles of asymmetric cell division along an invariant polarity axis instructed by

39 centrosomes. During interphase, the NSC centrosomes are defined by marked asymmetries in

40 protein composition and functional activity as microtubule-organizing centers. Here, we show a

41 conserved RNA-binding protein, Orb2, supports centrosome asymmetry in interphase NSCs.

42 While Orb2 localizes to the active apical centrosome, it promotes the transient inactivation of the

43 basal centrosome required for centrosome segregation and spindle morphogenesis. Orb2 is

44 required cell autonomously within NSCs to support centrosome asymmetry and maintenance of

45 the stem cell pool. Conversely, loss of orb2 manifests in microcephaly independent of Orb2

46 function in NSCs. We suggest Orb2 plays opposing roles in centrosome activation and

47 inactivation, possibly through the translational regulation of multiple mRNA substrates.

48 Bioinformatics uncovers a significant overlap among RNA targets between Drosophila Orb2 and

49 human CPEB4, consistent with a conserved role for CPEB proteins in centrosome regulation

50 and neurodevelopment.

\section{Introduction}

Neural stem cells (NSCs) undergo asymmetric cell division (ACD) along an invariant

56 apical-basal polarity axis to segregate cell fate determinants, giving rise to two differentially

57 fated progeny: a self-renewing stem cell and a ganglion mother cell (GMC) fated for neural

58 differentiation (Doe et al., 1991; Knoblich et al., 1995; Kraut et al., 1996; Broadus and Doe,

59 1997). This balance in NSC self-renewal is critical for neurogenesis, as its deregulation can lead

60 to brain tumors or neurodevelopmental disorders, such as microcephaly (Bond et al., 2002;

61 Cabernard and Doe, 2009). Key to NSC homeostasis are centrosomes, which instruct the 
62 division axis and organize the bipolar mitotic spindle required to segregate the pro-stem and

63 pro-differentiation cell fate determinants (Cabernard and Doe, 2009; Januschke and Gonzalez,

64 2010; Wang et al., 2011).

65 Centrosomes are microtubule (MT)-organizing centers (MTOC) consisting of a central

66 pair of centrioles surrounded by pericentriolar material (PCM), which recruits the $\gamma$-Tubulin

67 ( $\gamma$ Tub) ring complex required for MT nucleation (Conduit et al., 2015). Normally, centrosomes

68 recruit the robust levels of PCM necessary for microtubule-nucleating activity just before mitotic onset, a process called centrosome maturation (Gould and Borisy, 1977; Khodjakov and Rieder, 1999). Following mitotic exit, centrosomes shed PCM. In NSCs, however, centrosomes are subject to an asymmetric centrosome maturation

72 cycle, wherein the apical (daughter) centrosome recruits PCM and organizes MTs, while the 73 basal-fated (mother) centrosome is transiently inactivated until mitotic onset (Rebollo et al.,

74 2007; Rusan and Peifer, 2007; Conduit and Raff, 2010; Januschke et al., 2011). NSC

75 centrosome asymmetry is implicated in apical-basal spindle pole alignment and centrosome 76 segregation (Januschke and Gonzalez, 2010; Januschke et al., 2013; Lerit and Rusan, 2013;

77 Ramdas Nair et al., 2016). A basic molecular framework required for NSC centrosome

78 asymmetry involves asymmetric localization of Centrobin (Cnb) and Polo kinase to the daughter

79 centrosome in a mechanism also requiring Wdr62 to promote centrosome maturation

80 (Januschke et al., 2013; Ramdas Nair et al., 2016; Gallaud et al., 2020). Conversely, transient

81 inactivation of the basal centrosome requires Bld10/Cep135, Pericentrin-like protein (PLP), and

82 Polo-like kinase 4 (PLK4/SAK) (Lerit and Rusan, 2013; Singh et al., 2014; Gambarotto et al.,

83 2019). Nevertheless, how centrosome asymmetry is regulated remains incompletely

84 understood. 
87 study, raising the possibility that Orb2 might regulate centrosome asymmetry in NSCs (Stepien

88 et al., 2016). Orb2 is a member of the cytoplasmic polyadenylation element binding (CPEB)

89 proteins orthologous to mammalian CPEB2-4 and implicated in mRNA localization and

90 translational control (Huang et al., 2006; Keleman et al., 2007; Hafer et al., 2011). Although prior

91 work supports a role for orb2 in NSC spindle orientation and neuronal specification, whether

92 Orb2 contributes to centrosome regulation is unknown (Hafer et al., 2011).

93 Here, we identify an NSC-autonomous role for Orb2 in establishing centrosome

94 asymmetry associated with misaligned spindles and NSC loss. We also identify an NSC-

95 independent role for Orb2 in regulating brain size, as orb2 loss leads to microcephaly. Finally,

96 we examine potential targets of Orb2 and propose a revised model of asymmetric centrosome

97 maturation.

\section{Results and Discussion}

\section{Orb2 disrupts centrosome activity in interphase NSCs}

103 To determine if Orb2 contributes to centrosome asymmetry, we examined $\gamma$ Tub distributions at

104 apical and basal centrosomes in wild-type (WT) vs. orb2 null mutant NSCs during late

105 interphase, when centrosomes are normally asymmetric (Rebollo et al., 2007; Rusan and

106 Peifer, 2007). To directionally measure protein localization to centrosomes, we calculated an

107 asymmetry index ( $\mathrm{Al}$; Methods), wherein a value near 0 indicates symmetric distribution, while

108 values near 1 or -1 indicate asymmetric enrichment at the apical or basal centrosome,

109 respectively. Centrosomes visualized with the centriole marker Asterless (Asl; (Varmark et al.,

110 2007) displayed the expected symmetric distribution among apical and basal centrosomes in

111 both genotypes (mean $\mathrm{Al} \pm$ S.D. $=0.0 \pm 0.2$ for $\mathrm{WT}$ and $0.1 \pm 0.3$ for orb2; Fig. $1 \mathrm{~A}-\mathrm{C}$ ). In

112 comparison, $\gamma$ Tub was significantly enriched on the apical centrosome in WT NSCs (Fig. 1A, C). 
113 In contrast to WT, orb2 NSCs showed impaired centrosome asymmetry, evident by increased

$114 \gamma$ Tub localization to the basal centrosome and decreased Al values (Fig. 1B, C). $\gamma$ Tub Al was

115 reduced by over $20 \%$ within orb2 NSCs, as compared to WT (Fig 1C; $p=0.05$ by Kolmogorov-

116 Smirnov test). Consistently, $\sim 30 \%$ of orb2 NSCs $(N=12 / 40)$ had $\gamma$ Tub Al values $>2$ S.D. from

117 the WT mean (Fig 1D; $p<0.0001$ by chi-square test). Measuring the levels of $\gamma$ Tub localized at

118 the apical and basal centrosomes revealed a 2.7-fold increase in $\gamma$ Tub recruitment to the basal

119 centrosomes of symmetrized orb2 NSCs relative to WT (Fig. 1E; $p<0.0001$ by Mann-Whitney

120 test). We conclude Orb2 primarily promotes centrosome asymmetry during interphase by

121 blocking the precocious activation of the basal centrosome, although diminished recruitment of

$122 \gamma$ Tub to the apical centrosome may compound this effect.

124 Orb2-dependent centrosome regulation is cell autonomous

125 ACD is regulated through intrinsic and extrinsic cellular pathways (Siegrist and Doe, 2006; Doe, 126 2008). Prior work indicates Orb2 is broadly expressed throughout the larval central nervous 127 system (Keleman et al., 2007; Hafer et al., 2011). To elucidate if the reduction of centrosome 128 asymmetry observed in orb2 mutants arose from a requirement for Orb2 within NSCs, we 129 depleted orb2 specifically in NSCs using an orb2 dsRNA transgene (UAS-orb2 ${ }^{\text {RNAi }}$ ) driven by the 130 NSC-specific worniu (wor)-GAL4 (Albertson et al., 2004). Both the no GAL4 control and the wor$131 \mathrm{GAL4>orb2} 2^{R N A i}$ (hereafter, orb2 ${ }^{R N A i}$ ) interphase NSCs showed equal distributions of Asl at apical 132 and basal centrosomes (Fig. 1F-H). While controls appeared WT with an enrichment of $\gamma$ Tub on 133 the apical centrosome, a subset of orb2 ${ }^{R N A i} \mathrm{NSCs}(\sim 30 \% ; \mathrm{N}=16 / 51)$ recruited $\gamma \mathrm{Tub}$ to the basal

134 centrosome precociously and showed reduced $\gamma$ Tub at the apical centrosome (Fig. 1G-J).

135 These data indicate Orb2 promotes centrosome asymmetry cell autonomously within NSCs

136 through opposing activities at the apical and basal centrosomes. 


\section{Loss of orb2 is associated with supernumerary centrosomes}

139 The precocious activation of the basal centrosome is associated with errant centrosome

140 segregation during $A C D$, resulting in both centrosomes retained within the self-renewing stem

141 cell (Lerit and Rusan, 2013). To assay whether orb2 loss similarly impairs centrosome

142 segregation, we quantified the frequency of supernumerary centrosomes in WT and orb2 NSCs.

143 While $>90 \%$ WT NSCs $(\mathrm{N}=25 / 30)$ had the expected 2 centrosomes, $\sim 35 \%$ orb2 mutant NSCs

$144(\mathrm{~N}=15 / 40)$ had extra centrosomes (Fig 2A-C; $p<0.05$ by chi-square test). A similar frequency of

145 supernumerary centrosomes was observed in orb2 ${ }^{\text {RAAi }} \mathrm{NSCs}(\sim 25 \%$; $N=13 / 51 ; p<0.05$ by chi-

146 square test), demonstrating Orb2 functions within NSC to regulate centrosome number, likely

147 due to failed segregation of the basal centrosome to the GMC (Fig. 2D-F).

\section{Orb2 is required for mitotic spindle morphogenesis}

150 Given that Orb2 helps regulate centrosome activity and segregation, we next examined its role

151 in spindle orientation. During ACD, the NSC mitotic spindle normally orients along an invariant

152 apical-basal polarity axis entrained by the concerted action of multiple protein complexes

153 (Siegrist and Doe, 2005; Siller et al., 2006; Cabernard and Doe, 2009). Apical polarization is

154 initiated by the Par-complex, comprising Bazooka (Baz)/Par-3, Par-6, and atypical protein

155 kinase C ( $\alpha$ PKC; (Kuchinke et al., 1998; Cai et al., 2003; Goldstein and Macara, 2007)).

156 Inhibitory phosphorylation events by $\alpha$ PKC restrict basal fate determinants and the adapter

157 protein Miranda (Mira) to the basal cortex (Betschinger et al., 2003; Atwood and Prehoda,

158 2009).

We examined polarity establishment in mitotic WT and orb2 NSCs by monitoring the

160 localization of Baz and Mira. Localization of Baz to the apical cortex in late interphase initiates

161 NSC polarization (Wodarz and Huttner, 2003; Knoblich, 2008), while localization of Mira to the

162 basal cortex during mitosis represents a late polarization step (Shen et al., 1998; Rolls et al., 
2003; Atwood and Prehoda, 2009). WT and orb2 mutant NSCs showed similar distributions of

164 Baz and Mira to the apical and basal cortices, respectively (Fig 2G-I; n.s. by chi-square test).

165 These data argue that polarization is not significantly disrupted in orb2 larval NSCs.

We next used multiple coordinate analysis to define spindle orientation in WT and orb2

NSCs stained for $\beta$-Tubulin to label MTs, Baz, and Asl (see Methods; Fig. 2J-L). First, we measured spindle orientation $\left(\theta_{1}\right)$, the angle between the apical cortical polarity and spindle alignment axes (Fig. 2J, M-N). In WT, most spindles aligned within $30^{\circ}$ of the polarity axis, yet $\sim 20 \%$ of orb2 NSC spindles were misoriented $>30^{\circ}$, indicating orb2 spindles are more

171 randomized than controls (Fig $2 \mathrm{M}, \mathrm{N} ; p<0.0001$ by chi-square test).

174 anchoring of the apical centrosome to the apical cortex happens during early interphase and

175 influences polarization in NSCs and other stem cells (Yu et al., 2003; Januschke and Gonzalez,

176 2010; Inaba et al., 2015). Both WT and orb2 NSCs showed alignment of the apical centrosome

177 to the apical polarity axis, consistent with our observations that apical polarity is unaffected in

178 orb2 mutants (Fig. 2G-I, O-P).

Finally, we examined centrosome alignment, $\theta_{3}$, the angle between the apical and basal

180 centrosome axes (Fig 2J, Q-R). About $90 \%$ of WT mitotic NSCs aligned the basal centrosome

$181 \sim 180^{\circ}$ away from the apical centrosome (Fig $\left.2 \mathrm{~K}, \mathrm{Q}-\mathrm{R}\right)$. In contrast, $\theta_{3}$ is reduced in orb2 NSCs

182 (Fig. 2L, Q). While the average reduction in $\theta_{3}$ is not statistically significant, a subpopulation of

183 orb2 NSCs ( 20\%; N=7/32 cells) show defective $\theta_{3}<120^{\circ}$ (Fig. 2L, Q). Consequently, spindle

184 morphogenesis is significantly impaired, resulting in a higher frequency $(\sim 25 \%$; $N=9 / 34$ cells;

$185 p<0.0001$ by chi-square test) of bent spindles in orb2 NSCs relative to WT (Fig 2R). Taken

186 together, these data argue the precocious activation of the basal centrosome in orb2 NSCs

187 during interphase impairs centrosome migration to the basal cortex, resulting in aberrant spindle 
alignment and morphology. Alternatively, orb2 may have a distinct functions in interphase centrosome asymmetry and mitotic spindle orientation.

\section{Loss of orb2 results in microcephaly}

192 Defects in centrosome regulation contribute to neurodevelopmental disorders, including 193 microcephaly and intellectual disability (Robinson et al., 2020). Previous work illustrates orb2 is

194 crucial for learning and memory in the adult Drosophila brain (Keleman et al., 2007; Mastushita-

195 Sakai et al., 2010; Kruttner et al., 2012; Kacsoh et al., 2015; Hervas et al., 2016; Sanguanini

196 and Cattaneo, 2018). Moreover, reduced brain volumes were noted from serial sectioning adult

197 orb2 brains (Kruttner et al., 2012). Thus, to assess larval neurodevelopment in orb2 mutants, we

198 measured the volume of single optic lobes from age-matched third instar larva (Link et al.,

199 2019). Compared to WT brains, orb2 brains were significantly smaller (Fig $3 A, B, E ; p<0.0001$ by

200 t-test). About $75 \%(\mathrm{~N}=10 / 13$ cells) of orb2 brains had volumes < 2-S.D. from WT, consistent

201 with microcephaly.

To determine if NSC loss contributes to orb2-dependent microcephaly, we counted the

203 number of Mira+ NSCs in control and orb2 brains. While WT had 80 NSCs, orb2 mutants

204 showed a $\sim 20 \%$ reduction with $\sim 60$ NSCs per lobe (Fig 3C,D,F; $p<0.001$ by t-test). These data

205 indicate Orb2 is required to maintain the NSC pool.

Are the centrosome and spindle defects observed in orb2 mutants correlated with NSC

207 loss and/or microcephaly? To begin to address this question, we depleted orb2 specifically in

208 NSCs and compared the brain volume of age matched orb2 ${ }^{R N A i}$ brains relative to no GAL4

209 controls and noted no difference (Fig. $3 \mathrm{G}, \mathrm{H}, \mathrm{K}$ ), indicating orb2-dependent microcephaly is

210 nonautonomous to NSCs - Orb2 is required in other cell types to affect brain size. In contrast,

211 NSC loss is a cell autonomous response to orb2 depletion. Similar to orb2 mutants, we detected

$212 \sim 30 \%$ fewer NSC per optic lobe in orb2 ${ }^{R N A i}$ brains relative to no GAL4 controls (Fig 3I,J,L;

$213 p<0.0001$ by t-test). We conclude the frequency of NSC loss in orb2 mutants is similar to the 
214 incidence of centrosome defects, arguing these responses are correlated. Moreover, NSC loss

215 may be uncoupled from orb2-dependent microcephaly, consistent with the idea that Orb2

216 supports neurodevelopment in multiple cellular lineages.

217 To elucidate mechanisms underlying NSC loss in orb2 mutants, we first tested the

218 hypothesis that NSCs are eliminated by cell death. We quantified the coincidence of pro-

219 apoptotic cleaved Caspase 3 (CC3; (Fan and Bergmann, 2010)) in cells marked with the NSC-

220 specific Deadpan (Dpn) antibody (Bier et al., 1992). Similar rates of apoptosis were observed in

221 WT and orb2 NSCs, indicating NSC loss occurs by other mechanisms (Fig S1 A-C; n.s. by t-

222 test). Another mechanism whereby NSCs may be depleted is via premature differentiation

223 (Cabernard and Doe, 2009; Lai and Doe, 2014; Abdel-Salam et al., 2020). Normally, the pro-

224 differentiation marker Prospero (Pros) is confined to the nucleus of the differentiating GMCs and

225 absent from NSCs (Doe et al., 1991; Vaessin et al., 1991), while retention of Pros in the NSC

226 nucleus promotes premature differentiation (Lai and Doe, 2014). We quantified the coincidence

227 of Pros with Dpn and detected no significant difference relative to WT, indicating premature

228 differentiation does not precipitate NSC loss in orb2 mutants (Fig S1D-F; n.s. by t-test).

229 Finally, we examined whether orb2 affects mitotic progression, reasoning impaired NSC

230 self-renewal might contribute to NSC loss. However, the mitotic indices in WT and orb2 brains

231 were not significantly different $(33.6 \pm 4.9 \%$ per lobe in $\mathrm{N}=6$ WT vs. $36.3 \pm 10.1 \%$ in $\mathrm{N}=10$ orb2

232 brains; Fig S1G-I; n.s. by t-test). These data suggest NSC loss is not due to increased

233 quiescence. Loss of NSCs observed in orb2 mutants may be actuated by non-apoptotic cell

234 death pathways. Alternatively, the altered neuronal specification observed in orb2 embryos may

235 impinge upon larval neurodevelopment, resulting in fewer NSCs (Hafer et al., 2011).

237 Orb2 localizes to centrosomes within cycling NSCs

238 Our data indicate Orb2 functions autonomously within NSCs to regulate centrosome

239 asymmetry. Previous work indicates CPEB proteins localize to centrosomes in Xenopus 
240 embryos and cultured mammalian cells (Groisman et al., 2000; Pascual et al., 2020a; Pascual

241 et al., 2020b). Although Orb2 localizes to neuronal lineages, subcellular localization of Orb2 is

242 not well defined (Keleman et al., 2007; Hafer et al., 2011). Therefore, to inform mechanisms of

243 how Orb2 functions within NSCs to support centrosome asymmetry and spindle morphogenesis,

244 we examined endogenous Orb2 localization in NSCs using monoclonal antibodies (Hafer et al.,

245 2011). In interphase NSCs, Orb2 appeared dispersed throughout the cytoplasm with a notable

246 enrichment on the apical centrosome (Fig 4A, interphase). Apical centrosome enrichment of

247 Orb2 was observed in N=21/24 interphase NSCs, indicated by positive Orb2 Al values (Fig. 4B).

248 On average, interphase apical centrosomes contained 1.7-fold more Orb2 than basal

249 centrosomes (Fig. 4C; $p<0.01$ by t-test). Upon mitotic entry, Orb2 localization increased at both

250 centrosomes, demonstrating Orb2 is recruited to active centrosomes during centrosome

251 maturation (Fig 4A, prophase and metaphase). Our localization analysis reveals two populations

252 of Orb2 in interphase NSCs, a cytoplasmic versus centrosomal pool, raising the possibility that

253 Orb2 may normally function to regulate centrosome activity locally at centrosomes, from a

254 distance within the cytoplasm, or both.

257 Orb2 regulates PLP protein levels in larval brains

258 Because Orb2 is an RNA-binding protein known to promote or repress translation of its target

259 mRNAs, we reasoned it may contribute to centrosome regulation indirectly through regulation of

260 an mRNA associated with centrosome asymmetry (Mastushita-Sakai et al., 2010; Xu et al.,

261 2012; Khan et al., 2015). A genome-wide analysis for mRNA targets bound by Orb2 within

262 Drosophila S2 cell extracts uncovered thousands of putative targets, including mRNAs of genes

263 required for NSC centrosome asymmetry, e.g., Cep135, Cnb, plp, polo, and Wdr62 mRNAs

264 (Januschke et al., 2013; Lerit and Rusan, 2013; Singh et al., 2014; Ramdas Nair et al., 2016;

265 Stepien et al., 2016; Gallaud et al., 2020). While these transcripts were identified as potential 
Orb2 targets, they varied in their biologic complexity $(\mathrm{BC})$, a metric of reproducibility across biological replicates (Licatalosi et al., 2008).

Aspects of the orb2 null phenotype in NSCs resemble plp loss, as both mutants display precocious activation of the basal centrosome in interphase NSCs coincident with supernumerary centrosome and spindle defects (Lerit and Rusan, 2013). Given these

271 similarities, we tested if Orb2 regulates PLP protein expression by comparing PLP levels in WT

272 vs. orb2 larval brain extracts. Semi-quantitative western blotting uncovered an average $30 \%$

273 reduction in PLP in orb2 relative to WT, suggesting Orb2 promotes PLP translation (Fig. 5A, B).

274 To assay a requirement for Orb2 in regulating PLP in NSCs, we examined PLP localization to

275 the apical versus basal centrosomes in interphase NSCs. In WT NSCs, PLP was enriched on

276 the inactive, basal centrosome, consistent with prior work (Fig. 5C; (Lerit and Rusan, 2013;

277 Singh et al., 2014). Despite a reduction in PLP levels in whole brain extracts, robust localization

278 of PLP to centrosomes was detected in orb2 NSCs, comparable to WT (Fig. 5C, D; n.s. by

279 Kolmogorov-Smirnov test). Further, levels of PLP localized at apical vs. basal centrosomes

280 were not significantly different from WT (Fig. 5E). Taken together, these data imply Orb2-

281 dependent regulation of PLP may occur outside of NSCs and raise the possibility that other

282 Orb2 targets contribute to NSC centrosome regulation.

To refine the list of targets that may be involved in NSC centrosome regulation, we

284 compared the Orb2 mRNA targets identified in Drosophila S2 cells (Stepien et al., 2016) to a

285 recent list of mRNA targets bound by CPEB4 in HeLa cells (Pascual et al., 2020b) and identified

2861083 overlapping genes (Fig S2A; Table S1). Gene ontology (GO) analysis for cellular

287 components uncovered a significant enrichment of organelle terms (Fig S2B). In contrast,

288 centrosome-related ontologies were not significantly enriched among the overlapping genes

289 (Fig S2C). However, because Orb2 has multiple orthologs, CPEB2-4, other putative Orb2

290 targets absent from the CPEB4 dataset are omitted from these pairwise analyses. Therefore,

291 we examined centrosome-related ontologies of Orb2 targets with a $B C \geq 3$. This analysis 
292 identified 2150 mRNAs, among which 53 mRNAs were annotated with centrosome ontologies,

293 which may be subject to Orb2 regulation (Fig. S2D; Table S1).

295 Model Summary

296 Our data indicate Orb2 is required for robust inactivation of the basal centrosome and may also

297 enhance apical centrosome activity in interphase NSCs. Orb2 also functions in other cell types

298 for normal brain size (Fig 5F). Given its local enrichment, we propose Orb2 promotes apical

299 centrosome maturation (Fig 5F, top box), while cytoplasmic Orb2 inactivates the basal

300 centrosome, perhaps by repressing pro-maturation factors (Fig 5F, bottom box). Consistent with

301 opposing activities at the apical vs. basal centrosomes, Orb2 activates or represses the

302 translation of its targets depending on its oligomerization status ((Khan et al., 2015)). This model

303 predicts cytoplasmic Orb2 is predominantly monomeric, thereby functioning as a translational

304 repressor, while centrosome-localized Orb2 is oligomerized and functions as a translational

305 activator. Whether on-site translation occurs at the apical centrosome remains to be tested and

306 specific Orb2 targets require identification.

307 Microcephaly is quite heterogenous; however, a significant number of genes associated

308 with heritable microcephaly and intellectual disability are also associated with centrosome

309 biogenesis and regulation (Thornton and Woods, 2009; Jayaraman et al., 2018; Robinson et al., 310 2020). Our study implicates Orb2 at the intersection of these pathways. 


\section{Materials and Methods}

\section{Fly Stocks}

316 The following strains and transgenic lines were used: $y^{1} w^{1118}$ (Bloomington Drosophila Stock

317 Center (BDSC) \#1495) was used as the WT control unless otherwise noted. orb2 brains were

318 isolated from the homozygous null allele, orb2 ${ }^{436}$ (gift from P. Schedl, Princeton University (Xu

319 et al., 2012)). NSC-specific depletion of orb2 by orb2 ${ }^{R N A i}\left(P\{T R i P . H M J 22715\}^{\text {attP40; }}\right.$, BDSC

320 \#60424) was driven by ( $P\{\text { wor.GAL4.A }\}^{2}$; BDSC \#56555). All strains were maintained on

321 Bloomington formula cornmeal-agar media (Lab-Express, Inc.; Ann Arbor, $\mathrm{MI}$ ) at $25^{\circ} \mathrm{C}$ in a light

322 and temperature-controlled incubator.

Immunofluorescence

325 Crawling third instar larva were used for dissections. Larval brains were prepared for

326 immunofluorescence as previously described (Lerit et al., 2014). Briefly, brains were dissected

327 in Schneider's Drosophila Medium (ThermoFisher Scientific, \#21720024), fixed in 9\%

328 paraformaldehyde for 15 min, blocked in PBT buffer (Phosphate Buffered Saline (PBS)

329 supplemented with $1 \%$ BSA and $0.1 \%$ Tween-20) for one hour at room temperature prior to

330 overnight incubation in primary antibodies in PBT with nutation at $4^{\circ} \mathrm{C}$. Samples were further

331 blocked with modified PBT (2\% BSA, 0.1\% Tween-20, and 4\% normal goat serum in PBS) for 1

332 hour before incubation for two hours at room temperature with secondary antibody and DAPI.

333 Brains were oriented and mounted in Aqua-Poly/Mount (Polysciences, Inc) prior to imaging.

334 The following primary antibodies were used: guinea pig anti-Asl (1:4000, gift from G.

335 Rogers, University of Arizona), mouse anti-GTU88 ( $\gamma$ Tub; 1:250-350, Sigma T6557), rabbit anti-

336 Cnn (1:4000 gift from Tim Megraw, Florida State University), guinea pig anti-PLP (1:4000, gift

337 from Nasser Rusan, NIH), rabbit anti-phospho-Histone H3 Ser10 (pH3; 1:2000, Sigma-Millipore,

338 05-570), mouse anti- $\beta$-Tub (clone E7, 1:250; Developmental Studies Hybridoma Bank (DSHB)), 
mouse anti-Orb2 (undiluted 1:1 mix of clones 2D11 and 4G8 (DSHB); (Xu et al., 2012)), rat antiMira (1:500, Abcam, ab197788), rabbit anti-Baz (1:2000, gift from A. Harris, University of

341 Toronto), rat anti-Dpn (1:500, Abcam, ab195173), rabbit anti-cleaved Caspase 3 (CC3, 1:75;

342 Cell Signaling Technology, 9661s), and mouse anti-Pros (clone MR1A, 1:500; DSHB).

343 Secondary antibodies: Alexa Fluor 488, 568, and 647 (1:500, Molecular Probes). DAPI

344 (ThermoFisher Scientific) was used at $10 \mathrm{ng} / \mathrm{mL}$.

\section{Microscopy}

347 Images were acquired on a Nikon Ti-E inverted microscope fitted with a Yokogawa CSU-X1

348 spinning disk head (Yokogawa Corp. of America), Orca Flash 4.0 v2 CMOS camera

349 (Hamamatsu Corp.), Perfect Focus system (Nikon), and a Nikon LU-N4 solid-state laser launch

$350(15 \mathrm{~mW} ; 405,488,561$, and $647 \mathrm{~nm})$ using the following Nikon objectives: 100x 1.49-NA Apo

351 Total Internal Reflection Fluorescence oil immersion, 40x 1.3-NA Plan Fluor oil immersion, and

$3522 x \times 0.75-N A$ Plan Apo. Images were acquired at $25^{\circ} \mathrm{C}$ through Nikon Elements AR software on

353 a 64-bit HP Z440 workstation (Hewlett-Packard).

Image analysis

356 Images were assembled using Fiji (National Institutes of Health;(Schindelin et al., 2012)), Adobe

357 Photoshop, and Adobe Illustrator software to separate or merge channels, crop regions of

358 interest, generate maximum-intensity projections, and adjust brightness and contrast.

\section{Centrosome asymmetry}

361 Interphase NSCs were identified by the absence of $\mathrm{pH}$, round nuclear morphology, and

362 presence of duplicated centrosomes in large $(\geq 10 \mu \mathrm{m})$ cells. To blind the experimenter to

363 genotype, maximum projected images were generated, randomized, and used to measure

364 background-subtracted integrated densities from regions of interest (ROIs) centered at the 
apical or basal centrosomes. Apical centrosome integrated density $(A)$ and basal centrosome integrated density $(B)$ were used to calculate the asymmetry index, $(A-B) /(A+B)(($ Lerit and Rusan, 2013)). The data were then unblinded and mean \pm S.D. were calculated per genotype.

\section{Polarity, NSC number, and mitotic index}

370 Mitotic NSCs were identified by the presence of $\mathrm{pH} 3$ in large, Mira+ cells. To score polarity,

371 maximum projected images were anonymized to blind the experimenter to genotype, and each

372 NSC was scored for the absence or presence of Baz or Mira crescents at the apical or basal

373 cortices, respectively. All Mira+ NSCs were counted to calculate the number of NSCs per optic

374 lobe. Mitotic index is defined as the number of Mira+, pH3+ NSCs per total Mira+ NSCs.

\section{Spindle morphology}

377 Z-stack images of mitotic NSCs labeled with anti- $\beta$-Tub to label the mitotic spindle, Asl to mark

378 the centrioles, and Baz to label the apical polarity axis were randomized to blind the

379 experimenter to genotype. The point tool in Fiji was used to record the $\mathrm{X}, \mathrm{Y}$, and $\mathrm{Z}$ coordinates of

380 four ROls per cell: 1) center of the apical centrosome, 2) center of the basal centrosome, 3)

381 center of the Baz apical crescent, and 4) center of the DAPI+ condensed chromosomes. These

382 points were used to calculate four different vectors, and vector analysis was used to calculate

383 the angles between specified vectors. The following angles $(\theta)$ were calculated: $\theta_{1}=$ spindle

384 orientation, the angle between the vectors defined by points 1 and 2 (division axis) relative to

385 points 3 and 4 (polarity axis); $\theta_{2}=$ apical polarity alignment is the angle between the vectors

386 defined by points 3 and 4 (apical polarity axis) relative to points 1 and 4 (apical centrosome

387 axis); and $\theta_{3}=$ centrosome alignment is the angle between the vectors defined by points 1 and 4

388 (apical centrosome axis) relative to points 2 and 4 (basal centrosome axis). Angles that fell 
outside \pm 2 S.D. from the control mean were defined as defective. For spindle alignment, NSCs with spindle angles $>75^{\circ}$ were classified as orthogonal.

\section{Age-matched brain volume}

393 To age match larva, 20 female virgins and 10 males of the appropriate genotype were allowed

394 to seed a vial for $24 \mathrm{hrs}$. After removal of the adults, vials were incubated at $25^{\circ} \mathrm{C}$ until crawling

395 third instar larva emerged. orb2 null progeny showed a developmental delay of $24-48 \mathrm{hrs}$ as

396 compared to controls; null larva took $~ 7-8$ days to emerge. Age-matched crawling third instar

397 larva were dissected and prepared for immunofluorescence. The entire volume of the DAPI-

398 labeled brain was imaged. Imaris software (Oxford Instruments) was used to select an ROI of

399 the optic lobe and measure the volume using the 3D surfaces tool (Link et al., 2019).

\section{NSC differentiation and death rate}

402 NSCs were identified by the presence of Dpn. To score for premature differentiation, maximum

403 projected images of Dpn and Pros stained brains were randomized to blind the experimenter to 404 genotype, and an ROI of the central brain region was used to calculate the Pearson's

405 Correlation Coefficient on background-subtracted and automatic threshold-masked images

406 using the Coloc 2 plugin for Fiji (Schindelin et al., 2012). To score cell death, a similar analysis 407 was run on Dpn and CC3 stained brains. To assess specificity of overlapping signals for both 408 experiments, the red channel (Pros or CC3) was rotated $90^{\circ}$ clockwise, and colocalization was 409 remeasured.

\section{Immunoblotting}

412 Larval brain extracts were prepared from 20 crawling third instar larva dissected in Schneider's 413 medium, removed of imaginal discs, transferred to fresh media, then rinsed once in cold PBST. 414 Samples were homogenized on ice in $30 \mu \mathrm{L}$ of fresh PBST using a cordless motor and plastic, 
415 disposable homogenizer, supplemented with $20 \mu \mathrm{L} 5 \mathrm{x}$ SDS loading buffer, boiled for $10 \mathrm{~min}$ at

$41695^{\circ} \mathrm{C}$, then stored at $-20^{\circ} \mathrm{C}$ or resolved on a commercial $7.5 \%$ polyacrylamide gel (Bio-Rad,

$417 \# 4568023$. Proteins were transferred to a $0.2 \mu \mathrm{m}$ nitrocellulose membrane (GE Healthcare) by

418 wet transfer in a buffer containing $25 \mathrm{mM}$ Tris- $\mathrm{HCl}, \mathrm{pH} 7.6,192 \mathrm{mM}$ glycine, 10\% methanol, and

$4190.02 \%$ SDS at $4{ }^{\circ} \mathrm{C}$. Membranes were blocked in $5 \%$ dry milk in TBST (Tris-buffered saline,

$420 \quad 0.05 \%$ Tween-20), washed well with TBST, and incubated overnight at $4{ }^{\circ} \mathrm{C}$ with primary

421 antibodies. After washing with TBST, membranes were incubated for $1.5 \mathrm{hr}$ in secondary

422 antibodies diluted 1:5000 in TBST. Bands were visualized with Clarity ECL substrate (Bio-Rad,

423 1705061) on a Bio-Rad ChemiDoc imaging system.

424 The following primary antibodies were used: rabbit anti-PLP (1:4000, gift from Nasser

425 Rusan, NIH), guinea pig anti-Asl (1:10,000, gift from Greg Rogers, University of Arizona), and

426 mouse anti-Orb2 (1:25 dilution each of 2D11 and 4G8; DSHB, Paul Schedl, Princeton University

427 (Hafer et al., 2011)). Secondary antibodies: goat anti-mouse HRP (1:5000, ThermoFisher

428 \#31430), goat anti-rabbit HRP (1:5000, ThermoFisher \#31460), and goat anti-guinea pig HRP

429 (1:5000, ThermoFisher \#A18769). Densitometry was measured in Adobe Photoshop and

430 protein levels are normalized to the Asl loading control. Full-size, replicated blots are available

431 to view on FigShare: https://doi.org/10.6084/m9.figshare.17052722. The experiment was

432 repeated with three biological replicates per genotype.

\section{Bioinformatics}

435 Gene names were converted to FlyBase identifiers using the Flybase.org tool 'Query by

436 symbols'(Larkin et al., 2021). Overlapping genes were identified by the 'COUNT IF' function in

437 Excel and Venn diagrams were plotted in R-Studio. GO cellular component analysis was done 438 using the Panther statistical overrepresentation test (http://www.pantherdb.org/), and Fisher's 
exact test was used to generate an adjusted p-value, i.e., false discovery rate (FDR)(Mi et al., 2021)

\section{Statistical analysis}

443 Data were plotted and statistical analysis performed using Microsoft Excel, GraphPad Prism,

444 and RStudio software. Normality distributions were determined using a D'Agnostino-Pearson or

445 Shapiro-Wilk normality test. Goodness-of-Fit chi-squared analysis were performed using control

446 distributions as the expected distributions. Data were further analyzed using parametric two-

447 tailed t-test, non-parametric Mann-Whitney test or Kolmogorov-Smirnov (KS) test, or an

448 appropriate post-hoc test as noted in the figure legends. Data are plotted as mean \pm S.D. and

449 are representative results from at 2 or more independent experiments.

\section{Supplemental materials}

Fig. S1 NSC differentiation and survival in WT vs. orb2 mutants. Maximum intensity projections of (A) WT and (B) orb2 brains stained with Dpn (green; NSC nuclei) and CC3 (red; pro-apototic. Insets (boxes) are enlarged to the right to highlight NSCs (dashed ovals). (C)

458 Quantification of the Pearson's correlation coefficient between Dpn and CC3 from N=10 WT and 45915 orb2 optic lobes pooled from two replicates. The CC3 channel was rotated clockwise $\left(90^{\circ}\right)$ to 460 test for specificity of overlapping signals. (D) Maximum intensity projections of WT and (E) orb2 461 brains stained with Dpn (green) and Pros (red; differentiated nuclei). (F) Quantification of the 462 Pearson's correlation coefficient between Dpn and Pros from N=16 WT and 18 orb2 optic lobes 463 pooled from two replicates. The Pros channel was rotated clockwise $\left(90^{\circ}\right)$ to test for specificity 464 of overlapping signals. In (D) and (F), each data point is from one ROI per optic lobe. (G) 
Projected images of WT and (H) orb2 NSCs (dashed ovals) stained for Mira (green; NSCs) and the mitotic marker $\mathrm{pH} 3$ (red). (I) Quantification of mitotic index from N=6 WT and 10 orb2 brains. n.s., not significant by Student's t-test. Bars: (A-E) $20 \mu \mathrm{m}$ and $8 \mu \mathrm{m}$ (insets); (G and H) $10 \mu \mathrm{m}$.

Fig. S2 Ontological analysis of Orb2 and human CPEB4. A) Venn diagram showing relative

470 transcript pool sizes and overlap detected from the Stepien et al. Orb2 CLIP and Pascual et al.

471 CPEB4 datasets (Stepien et al., 2016; Pascual et al., 2020b). B) Top 5 gene ontologies

472 overrepresented in the overlapping transcripts by lowest raw $p$ value. C) Overrepresentation

473 tests for gene ontologies implicated in the centrosome were not significant. D)

474 Overrepresentation test of genes in the Orb2 CLIPseq with BC $\geq 3$ yields significant centrosome 475 ontologies. - $\log p$-values are plotted with a significance cut-off of $p=0.05$, noted by the dashed 476 line and asterisk. Genes are listed in Table S1.

Table S1. Orb2 and CPEB4 common RNA targets. Sheet 1 lists FlyBase IDs for all transcripts

479 detected in (Stepien et al., 2016; Pascual et al., 2020b). Sheet 2 lists overlapping genes;

480 column $\mathrm{E}$ is sortable to list genes with centrosome ontologies. Sheet 3 lists GO terms

481 overrepresented in the overlapping genes, column $\mathrm{J}$ is sortable to list genes with centrosome

482 ontologies. Sheet 4 lists Orb2 targets from (Stepien et al., 2016) with $B C \geq 3$; column $F$ is

483 sortable to list genes with centrosome ontologies. Sheet 5 shows an overrepresentation test for 484 centrosome ontologies from the Orb2 $\mathrm{BC} \geq 3$ gene list. Sheet 6 lists each gene included in the 485 indicated centrosome ontologies.

\section{Competing interest statement}

489 The authors have no competing interests to declare. 


\section{Acknowledgements}

We thank Drs. Timothy Megraw, Nasser Rusan, Greg Rogers, and Paul Schedl for gifts of reagents. We are grateful to members of the Lerit lab and Drs. Victor Faundez and Nasser

494 Rusan for constructive feedback on the manuscript.

495 This work was supported by NIH grant T32GM008490 (BVR), American Heart Association

496 grant 20POST35210023 (to JF), and R01GM138544 (to DAL).

\section{Author contributions}

499 Beverly V. Robinson- data curation, formal analysis, funding acquisition, investigation,

500 methodology, visualization, writing-original draft, and writing-review \& editing.

501 Junnan Fang- formal analysis, funding acquisition, investigation, methodology, and writing-

502 review \& editing.

503 Dipen S. Mehta- investigation

504 Joseph D. Buehler- formal analysis, software, visualization, and writing-review \& editing.

505 Dorothy A. Lerit- conceptualization, formal analysis, funding acquisition, investigation,

506 methodology, project administration, supervision, visualization, writing-original draft, and

507 writing-review \& editing.

508

509

510 


\section{References}

513

514

515

516

517

518

519

520

521

522

523

524

525

526

527

528

529

530

531

532

533

534

535

536

537

538

539

540

541

542

543

544

545

546

547

548

549

550

551

552

553

554

555

556

557

558

559

560

561

Abdel-Salam, G.M.H., I.S.M. Sayed, H.H. Afifi, S.F. Abdel-Ghafar, M.R. Abouzaid, S.I. Ismail, M.S. Aglan, M.Y. Issa, H.T. El-Bassyouni, G. El-Kamah, L.K. Effat, M. Eid, M.S. Zaki, S.A. Temtamy, and M.S. Abdel-Hamid. 2020. Microcephalic osteodysplastic primordial dwarfism type II: Additional nine patients with implications on phenotype and genotype correlation. Am. J. Med. Genet. A. 182:1407-1420.

Albertson, R., C. Chabu, A. Sheehan, and C.Q. Doe. 2004. Scribble protein domain mapping reveals a multistep localization mechanism and domains necessary for establishing cortical polarity. J. Cell Sci. 117:6061-6070.

Atwood, S.X., and K.E. Prehoda. 2009. aPKC phosphorylates Miranda to polarize fate determinants during neuroblast asymmetric cell division. Curr. Biol. 19:723-729.

Betschinger, J., K. Mechtler, and J.A. Knoblich. 2003. The Par complex directs asymmetric cell division by phosphorylating the cytoskeletal protein Lgl. Nature. 422:326-330.

Bier, E., H. Vaessin, S. Younger-Shepherd, L.Y. Jan, and Y.N. Jan. 1992. deadpan, an essential pan-neural gene in Drosophila, encodes a helix-loop-helix protein similar to the hairy gene product. Genes Dev. 6:2137-2151.

Bond, J., E. Roberts, G.H. Mochida, D.J. Hampshire, S. Scott, J.M. Askham, K. Springell, M. Mahadevan, Y.J. Crow, A.F. Markham, C.A. Walsh, and C.G. Woods. 2002. ASPM is a major determinant of cerebral cortical size. Nat. Genet. 32:316-320.

Broadus, J., and C.Q. Doe. 1997. Extrinsic cues, intrinsic cues and microfilaments regulate asymmetric protein localization in Drosophila neuroblasts. Curr. Biol. 7:827-835.

Cabernard, C., and C.Q. Doe. 2009. Apical/basal spindle orientation is required for neuroblast homeostasis and neuronal differentiation in Drosophila. Dev. Cell. 17:134-141.

Cai, Y., F. Yu, S. Lin, W. Chia, and X. Yang. 2003. Apical complex genes control mitotic spindle geometry and relative size of daughter cells in Drosophila neuroblast and pl asymmetric divisions. Cell. 112:51-62.

Conduit, P.T., and J.W. Raff. 2010. Cnn dynamics drive centrosome size asymmetry to ensure daughter centriole retention in Drosophila neuroblasts. Curr. Biol. 20:2187-2192.

Conduit, P.T., A. Wainman, and J.W. Raff. 2015. Centrosome function and assembly in animal cells. Nat. Rev. Mol. Cell Biol. 16:611-624.

Doe, C.Q. 2008. Neural stem cells: balancing self-renewal with differentiation. Development. 135:1575-1587.

Doe, C.Q., Q. Chu-LaGraff, D.M. Wright, and M.P. Scott. 1991. The prospero gene specifies cell fates in the Drosophila central nervous system. Cell. 65:451-464.

Fan, Y., and A. Bergmann. 2010. The cleaved-Caspase-3 antibody is a marker of Caspase-9like DRONC activity in Drosophila. Cell Death Differ. 17:534-539.

Gallaud, E., A. Ramdas Nair, N. Horsley, A. Monnard, P. Singh, T.T. Pham, D. Salvador Garcia, A. Ferrand, and C. Cabernard. 2020. Dynamic centriolar localization of Polo and Centrobin in early mitosis primes centrosome asymmetry. PLoS Biol. 18:e3000762.

Gambarotto, D., C. Pennetier, J.M. Ryniawec, D.W. Buster, D. Gogendeau, A. Goupil, M. Nano, A. Simon, D. Blanc, V. Racine, Y. Kimata, G.C. Rogers, and R. Basto. 2019. Plk4 Regulates Centriole Asymmetry and Spindle Orientation in Neural Stem Cells. Dev. Cell. 50:11-24 e10.

Goldstein, B., and I.G. Macara. 2007. The PAR proteins: fundamental players in animal cell polarization. Dev. Cell. 13:609-622.

Gould, R.R., and G.G. Borisy. 1977. The pericentriolar material in Chinese hamster ovary cells nucleates microtubule formation. J Cell Biol. 73:601-615.

Groisman, I., Y.S. Huang, R. Mendez, Q. Cao, W. Theurkauf, and J.D. Richter. 2000. CPEB, maskin, and cyclin B1 mRNA at the mitotic apparatus: implications for local translational control of cell division. Cell. 103:435-447. 
562

563

564

565

566

567

568

569

570

571

572

573

574

575

576

577

578

579

580

581

582

583

584

585

586

587

588

589

590

591

592

593

594

595

596

597

598

599

600

601

602

603

604

605

606

607

608

609

610

611

612

Hafer, N., S. Xu, K.M. Bhat, and P. Schedl. 2011. The Drosophila CPEB protein Orb2 has a novel expression pattern and is important for asymmetric cell division and nervous system function. Genetics. 189:907-921.

Hervas, R., L. Li, A. Majumdar, C. Fernandez-Ramirez Mdel, J.R. Unruh, B.D. Slaughter, A. Galera-Prat, E. Santana, M. Suzuki, Y. Nagai, M. Bruix, S. Casas-Tinto, M. Menendez, D.V. Laurents, K. Si, and M. Carrion-Vazquez. 2016. Molecular Basis of Orb2 Amyloidogenesis and Blockade of Memory Consolidation. PLoS Biol. 14:e1002361.

Huang, Y.S., M.C. Kan, C.L. Lin, and J.D. Richter. 2006. CPEB3 and CPEB4 in neurons: analysis of RNA-binding specificity and translational control of AMPA receptor GluR2 mRNA. EMBO J. 25:4865-4876.

Inaba, M., Z.G. Venkei, and Y.M. Yamashita. 2015. The polarity protein Baz forms a platform for the centrosome orientation during asymmetric stem cell division in the Drosophila male germline. Elife. 4.

Januschke, J., and C. Gonzalez. 2010. The interphase microtubule aster is a determinant of asymmetric division orientation in Drosophila neuroblasts. J Cell Biol. 188:693-706.

Januschke, J., S. Llamazares, J. Reina, and C. Gonzalez. 2011. Drosophila neuroblasts retain the daughter centrosome. Nat Commun. 2:243.

Januschke, J., J. Reina, S. Llamazares, T. Bertran, F. Rossi, J. Roig, and C. Gonzalez. 2013. Centrobin controls mother-daughter centriole asymmetry in Drosophila neuroblasts. Nat. Cell Biol. 15:241-248.

Jayaraman, D., B.I. Bae, and C.A. Walsh. 2018. The Genetics of Primary Microcephaly. Annu Rev Genomics Hum Genet. 19:177-200.

Kacsoh, B.Z., J. Bozler, S. Hodge, M. Ramaswami, and G. Bosco. 2015. A novel paradigm for nonassociative long-term memory in Drosophila: predator-induced changes in oviposition behavior. Genetics. 199:1143-1157.

Keleman, K., S. Kruttner, M. Alenius, and B.J. Dickson. 2007. Function of the Drosophila CPEB protein Orb2 in long-term courtship memory. Nat. Neurosci. 10:1587-1593.

Khan, M.R., L. Li, C. Perez-Sanchez, A. Saraf, L. Florens, B.D. Slaughter, J.R. Unruh, and K. Si. 2015. Amyloidogenic Oligomerization Transforms Drosophila Orb2 from a Translation Repressor to an Activator. Cell. 163:1468-1483.

Khodjakov, A., and C.L. Rieder. 1999. The sudden recruitment of gamma-tubulin to the centrosome at the onset of mitosis and its dynamic exchange throughout the cell cycle, do not require microtubules. J Cell Biol. 146:585-596.

Knoblich, J.A. 2008. Mechanisms of asymmetric stem cell division. Cell. 132:583-597.

Knoblich, J.A., L.Y. Jan, and Y.N. Jan. 1995. Asymmetric segregation of Numb and Prospero during cell division. Nature. 377:624-627.

Kraut, R., W. Chia, L.Y. Jan, Y.N. Jan, and J.A. Knoblich. 1996. Role of inscuteable in orienting asymmetric cell divisions in Drosophila. Nature. 383:50-55.

Kruttner, S., B. Stepien, J.N. Noordermeer, M.A. Mommaas, K. Mechtler, B.J. Dickson, and K. Keleman. 2012. Drosophila CPEB Orb2A mediates memory independent of Its RNAbinding domain. Neuron. 76:383-395.

Kuchinke, U., F. Grawe, and E. Knust. 1998. Control of spindle orientation in Drosophila by the Par-3-related PDZ-domain protein Bazooka. Curr. Biol. 8:1357-1365.

Lai, S.L., and C.Q. Doe. 2014. Transient nuclear Prospero induces neural progenitor quiescence. Elife. 3.

Larkin, A., S.J. Marygold, G. Antonazzo, H. Attrill, G. Dos Santos, P.V. Garapati, J.L. Goodman, L.S. Gramates, G. Millburn, V.B. Strelets, C.J. Tabone, J. Thurmond, and C. FlyBase. 2021. FlyBase: updates to the Drosophila melanogaster knowledge base. Nucleic Acids Res. 49:D899-D907.

Lerit, D.A., K.M. Plevock, and N.M. Rusan. 2014. Live imaging of Drosophila larval neuroblasts. $J$ Vis Exp. 
613

614

615

616

617

618

619

620

621

622

623

624

625

626

627

628

629

630

631

632

633

634

635

636

637

638

639

640

641

642

643

644

645

646

647

648

649

650

651

652

653

654

655

656

657

658

659

660

661

662

Lerit, D.A., and N.M. Rusan. 2013. PLP inhibits the activity of interphase centrosomes to ensure their proper segregation in stem cells. J Cell Biol. 202:1013-1022.

Licatalosi, D.D., A. Mele, J.J. Fak, J. Ule, M. Kayikci, S.W. Chi, T.A. Clark, A.C. Schweitzer, J.E. Blume, X. Wang, J.C. Darnell, and R.B. Darnell. 2008. HITS-CLIP yields genome-wide insights into brain alternative RNA processing. Nature. 456:464-469.

Link, N., H. Chung, A. Jolly, M. Withers, B. Tepe, B.R. Arenkiel, P.S. Shah, N.J. Krogan, H. Aydin, B.B. Geckinli, T. Tos, S. Isikay, B. Tuysuz, G.H. Mochida, A.X. Thomas, R.D. Clark, G.M. Mirzaa, J.R. Lupski, and H.J. Bellen. 2019. Mutations in ANKLE2, a ZIKA Virus Target, Disrupt an Asymmetric Cell Division Pathway in Drosophila Neuroblasts to Cause Microcephaly. Dev. Cell. 51:713-729 e716.

Mastushita-Sakai, T., E. White-Grindley, J. Samuelson, C. Seidel, and K. Si. 2010. Drosophila Orb2 targets genes involved in neuronal growth, synapse formation, and protein turnover. Proc. Natl. Acad. Sci. U. S. A. 107:11987-11992.

Mi, H., D. Ebert, A. Muruganujan, C. Mills, L.P. Albou, T. Mushayamaha, and P.D. Thomas. 2021. PANTHER version 16: a revised family classification, tree-based classification tool, enhancer regions and extensive API. Nucleic Acids Res. 49:D394-D403.

Pascual, R., J. Martin, F. Salvador, O. Reina, V. Chanes, A. Millanes-Romero, C. Suner, G. Fernandez-Miranda, A. Bartomeu, Y.S. Huang, R.R. Gomis, and R. Mendez. 2020a. The RNA binding protein CPEB2 regulates hormone sensing in mammary gland development and luminal breast cancer. Sci Adv. 6:eaax3868.

Pascual, R., C. Segura-Morales, M. Omerzu, N. Bellora, E. Belloc, C.L. Castellazzi, O. Reina, E. Eyras, M.M. Maurice, A. Millanes-Romero, and R. Mendez. 2020b. mRNA spindle localization and mitotic translational regulation by CPEB1 and CPEB4. RNA.

Ramdas Nair, A., P. Singh, D. Salvador Garcia, D. Rodriguez-Crespo, B. Egger, and C. Cabernard. 2016. The Microcephaly-Associated Protein Wdr62/CG7337 Is Required to Maintain Centrosome Asymmetry in Drosophila Neuroblasts. Cell Rep. 14:1100-1113.

Rebollo, E., P. Sampaio, J. Januschke, S. Llamazares, H. Varmark, and C. Gonzalez. 2007. Functionally unequal centrosomes drive spindle orientation in asymmetrically dividing Drosophila neural stem cells. Dev. Cell. 12:467-474.

Robinson, B.V., V. Faundez, and D.A. Lerit. 2020. Understanding microcephaly through the study of centrosome regulation in Drosophila neural stem cells. Biochem. Soc. Trans. 48:2101-2115.

Rolls, M.M., R. Albertson, H.P. Shih, C.Y. Lee, and C.Q. Doe. 2003. Drosophila aPKC regulates cell polarity and cell proliferation in neuroblasts and epithelia. J Cell Biol. 163:1089-1098.

Rusan, N.M., and M. Peifer. 2007. A role for a novel centrosome cycle in asymmetric cell division. J Cell Biol. 177:13-20.

Sanguanini, M., and A. Cattaneo. 2018. A continuous model of physiological prion aggregation suggests a role for Orb2 in gating long-term synaptic information. $R$ Soc Open Sci. 5:180336.

Schindelin, J., I. Arganda-Carreras, E. Frise, V. Kaynig, M. Longair, T. Pietzsch, S. Preibisch, C. Rueden, S. Saalfeld, B. Schmid, J.Y. Tinevez, D.J. White, V. Hartenstein, K. Eliceiri, P. Tomancak, and A. Cardona. 2012. Fiji: an open-source platform for biological-image analysis. Nat Methods. 9:676-682.

Shen, C.P., J.A. Knoblich, Y.M. Chan, M.M. Jiang, L.Y. Jan, and Y.N. Jan. 1998. Miranda as a multidomain adapter linking apically localized Inscuteable and basally localized Staufen and Prospero during asymmetric cell division in Drosophila. Genes Dev. 12:1837-1846.

Siegrist, S.E., and C.Q. Doe. 2005. Microtubule-induced Pins/Galphai cortical polarity in Drosophila neuroblasts. Cell. 123:1323-1335.

Siegrist, S.E., and C.Q. Doe. 2006. Extrinsic cues orient the cell division axis in Drosophila embryonic neuroblasts. Development. 133:529-536. 

regulates spindle orientation in Drosophila neuroblasts. Nat. Cell Biol. 8:594-600.

Singh, P., A. Ramdas Nair, and C. Cabernard. 2014. The centriolar protein Bld10/Cep135 is required to establish centrosome asymmetry in Drosophila neuroblasts. Curr. Biol. 24:1548-1555.

Stepien, B.K., C. Oppitz, D. Gerlach, U. Dag, M. Novatchkova, S. Kruttner, A. Stark, and K. Keleman. 2016. RNA-binding profiles of Drosophila CPEB proteins Orb and Orb2. Proc. Natl. Acad. Sci. U. S. A. 113:E7030-E7038.

Thornton, G.K., and C.G. Woods. 2009. Primary microcephaly: do all roads lead to Rome? Trends Genet. 25:501-510.

Vaessin, H., E. Grell, E. Wolff, E. Bier, L.Y. Jan, and Y.N. Jan. 1991. prospero is expressed in neuronal precursors and encodes a nuclear protein that is involved in the control of axonal outgrowth in Drosophila. Cell. 67:941-953.

Varmark, H., S. Llamazares, E. Rebollo, B. Lange, J. Reina, H. Schwarz, and C. Gonzalez. 2007. Asterless is a centriolar protein required for centrosome function and embryo development in Drosophila. Curr. Biol. 17:1735-1745.

Wang, C., S. Li, J. Januschke, F. Rossi, Y. Izumi, G. Garcia-Alvarez, S.S. Gwee, S.B. Soon, H.K. Sidhu, F. Yu, F. Matsuzaki, C. Gonzalez, and H. Wang. 2011. An ana2/ctp/mud complex regulates spindle orientation in Drosophila neuroblasts. Dev. Cell. 21:520-533.

Wodarz, A., and W.B. Huttner. 2003. Asymmetric cell division during neurogenesis in Drosophila and vertebrates. Mech. Dev. 120:1297-1309.

Xu, S., N. Hafer, B. Agunwamba, and P. Schedl. 2012. The CPEB protein Orb2 has multiple functions during spermatogenesis in Drosophila melanogaster. PLoS Genet. 8:e1003079.

Yu, F., Y. Cai, R. Kaushik, X. Yang, and W. Chia. 2003. Distinct roles of Galphai and Gbeta13F subunits of the heterotrimeric $\mathrm{G}$ protein complex in the mediation of Drosophila neuroblast asymmetric divisions. J Cell Biol. 162:623-633. 


\section{Figure Legends}

Figure 1. Orb2 contributes to centrosome asymmetry. Maximum intensity projections of

722

723 interphase NSCs (dashed circles) stained for Asl (centrioles, magenta) and $\gamma$ Tub (PCM, cyan). Solid and dashed boxes mark apical vs. basal centrosomes; enlarged at right. (A) WT NSC with $\gamma$ Tub enriched at the apical centrosome. (B) orb2 NSC with $\gamma$ Tub at both centrosomes. (C) Als of Asl and $\gamma$ Tub in $\mathrm{N}=30$ NSCs from $n=8$ WT brains and $\mathrm{N}=40$ NSCs from $\mathrm{n}=10$ orb2 brains.

$724 \gamma$ Tub levels at apical vs. basal centrosomes in $\mathrm{N}=24 \mathrm{NSC}$ from $\mathrm{n}=8 \mathrm{WT}$ brains and $\mathrm{N}=10$ symmetrized NSCs from $\mathrm{n}=7$ orb2 brains (defined as $\mathrm{Al}>2$ S.D. from WT mean). (F) no GAL4

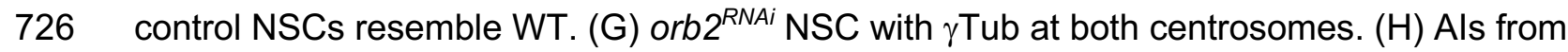

$727 \mathrm{~N}=39 \mathrm{NSC}$ from $\mathrm{n}=6$ no GAL4 brains and $\mathrm{N}=51 \mathrm{NSC}$ from $\mathrm{n}=6$ orb $2^{R N A i}$ brains. (I) Frequency 
728 distribution of Asl and $\gamma$ Tub Als in control vs. orb2 $2^{R N A i}$ brains. (J) Scatter plot of $\gamma$ Tub at

729 centrosomes from $\mathrm{N}=39$ NSCs from $n=6$ control brains and $\mathrm{N}=16$ NSCs from $n=6$

730 worGAL4>orb2 ${ }^{R N A i}$ brains. Mean \pm SD displayed. The experiments were repeated $\geq 3$

731 independent replicates and significance determined by $(C, H)$ Kolmogorov-Smirnov test, $(D, I)$

732 chi-square test, $(E, J)$ Mann-Whitney test: n.S., not significant; ${ }^{*}, p \leq 0.05 ;{ }^{* *} p \leq 0.01{ }^{* * *}, p<0.001$;

733 and $^{* * * *}, p<0.0001$. Bars: $5 \mu \mathrm{m}, 1 \mu \mathrm{m}$ (insets).

734

735 Figure 2. Orb2 is required for centrosome segregation and spindle morphogenesis.

736 Maximum intensity projections of interphase NSCs (dashed circles) from (A) WT, (B) orb2, (D)

737 no GAL4 control, and (E) orb2 ${ }^{R N A i}$ stained for Asl (arrows). Solid and dashed boxes mark apical

738 vs. basal centrosomes; enlarged at right. ( $C$ and F) Frequency distributions of supernumerary

739 centrosomes in interphase NSCs. (G) WT and (H) orb2 NSCs stained for Baz (apical cortex,

740 cyan), pH3 (mitotic, cyan), Mira (basal cortex, magenta), and Asl (yellow; arrowheads). (I)

741 Frequency distribution of apical Baz and/or basal Mira crescents in mitotic NSCs. (J) Cartoon

742 depicts points used to measure within mitotic NSCs: $\theta_{1}$ spindle orientation, $\theta_{2}$ apical polarity

743 alignment, and $\theta_{3}$ centrosome alignment (Methods). (K) WT and (L) orb2 NSCs stained for MTs

744 ( $\beta$-Tub, magenta), Asl (yellow), Baz (cyan), and DAPI (DNA, grey). (M) Plot and (N) frequency

745 distribution of $\theta_{1}$ spindle orientation. $(\mathrm{O})$ Plot and $(\mathrm{P})$ frequency distribution of $\theta_{2}$ apical polarity

746 alignment. (Q) Plot and (R) frequency distribution of $\theta_{3}$ centrosome alignment from N=33 WT

747 and $\mathrm{N}=32$ orb2 mitotic NSCs from a single experiment across two replicates. Light grey $\theta$ are $>2$

748 SD from the WT mean. Mean \pm SD indicated; significance determined by $(C, F, I, N, P, R)$ Chi-

749 squared test or $(\mathrm{M}, \mathrm{O}, \mathrm{Q})$ Two-tailed t-test. n.s., not significant; ${ }^{*}, p<0.05$; and ${ }^{* * * *}, p<0.0001$.

750 Bars: $5 \mu \mathrm{m} ; 1 \mu \mathrm{m}$ (insets). 
Figure 3. NSC-autonomous and non-autonomous Orb2 activities support

neurodevelopment. Projected third instar larval optic lobes (dashed lines) stained for DAPI or

Mira. In age-matched brains, (A) WT are larger than (B) orb2 and (C) WT have more NSCs than

(D) orb2. (E) Volume quantified from N=15 WT and 13 orb2 brains. (F) Quantification of NSCs in

$\mathrm{N}=6 \mathrm{WT}$ and 10 orb2 brains. In age-matched brains, $(\mathrm{G})$ no GAL4 controls are sized as $(\mathrm{H})$

757 orb2 ${ }^{R N A i}$ brains. Yet, (I) no GAL4 controls have more NSCs than (J) orb2 ${ }^{R N A i}$. (K) Volume and (L)

758 NSC counts quantified from N=6 brains per genotype. Brain volumes were measured from one

759 lobe per brain and normalized to the control mean (Link et al., 2019). Mean \pm SD displayed.

760 Significance determined by Student's t-test: n.s., not significant; ${ }^{*}, p<0.05^{\star * *}$; and $p<0.001$.

$761 \quad$ Bars: $40 \mu \mathrm{m}$.

762

763

Figure 4. Orb2 localizes to active NSC centrosomes. (A) Maximum-intensity projections of

764 WT NSCs (dashed circles) stained for Asl (magenta), Cnn (PCM; yellow), and Orb2 (cyan).

765 Solid and dashed boxes note apical vs. basal centrosomes; enlarged at right. (B) Orb2 Al and

766 (C) Orb2 levels on apical vs. basal centrosomes quantified from N=24 late interphase NSCs.

767 Mean \pm SD displayed; ${ }^{* *}, p=0.003$ by Welch's t-test. Bars: $5 \mu \mathrm{m}, 1 \mu \mathrm{m}$ (inset).

768

769 Figure 5. Orb2 regulates PLP protein levels. (A) Immunoblot of PLP, Asl, and Orb2 proteins

770 from WT and orb2 third instar larval brain extracts. (B) Normalized (to Asl) relative PLP levels in

771 WT and orb2 larval brains from 3 biological replicates. (C) Max projected WT and orb2 NSCs

772 (dashed circles) stained for $\gamma$ Tub (magenta) and PLP (cyan). Solid and dashed boxes note

773 apical vs. basal centrosomes; enlarged at right. (D) PLP Al calculated from N=24 WT and 32

774 orb2 interphase NSCs. (E) PLP localization to apical and basal centrosomes in N=24 WT vs.

$775 \mathrm{~N}=43$ orb2 interphase NSCs. Data representative from a single experiment across 2 replicates.

776 (F) Model depicts NSC-autonomous function of Orb2 to support interphase centrosome

777 asymmetry and NSC-independent requirement for normal brain volume. Loss of orb2 activity 
778 (grey cells) impairs basal centrosome inactivation (PCM, green). Centrosome-localized Orb2

779 (WT apical; top box) enhances centrosome activation, perhaps by translational activation of one

780 or more targets (black, Orb2 target $\mathrm{BC} \geq 3$; see Table S1). Conversely, cytoplasmic Orb2

781 supports basal centrosome inactivation (bottom box), required for centrosome separation,

782 spindle orientation, and centrosome segregation to the daughter cells. Mean \pm SD displayed; *,

$783 p<0.05$ by unpaired, one-tailed t-test; n.s., not significant. Uncropped, replicated blots are

784 available to view: https://doi.org/10.6084/m9.figshare.17052722 Bars: $5 \mu \mathrm{m}$; insets $1 \mu \mathrm{m}$. 
bioRxiv preprint doi: https://doi.org/10.1101/2021.11.23.469707; this version posted November 23, 2021. The copyright holder for this preprint (which was not certified by peer review) is the author/funder, who has granted bioRxiv a license to display the preprint in perpetuity. It is made
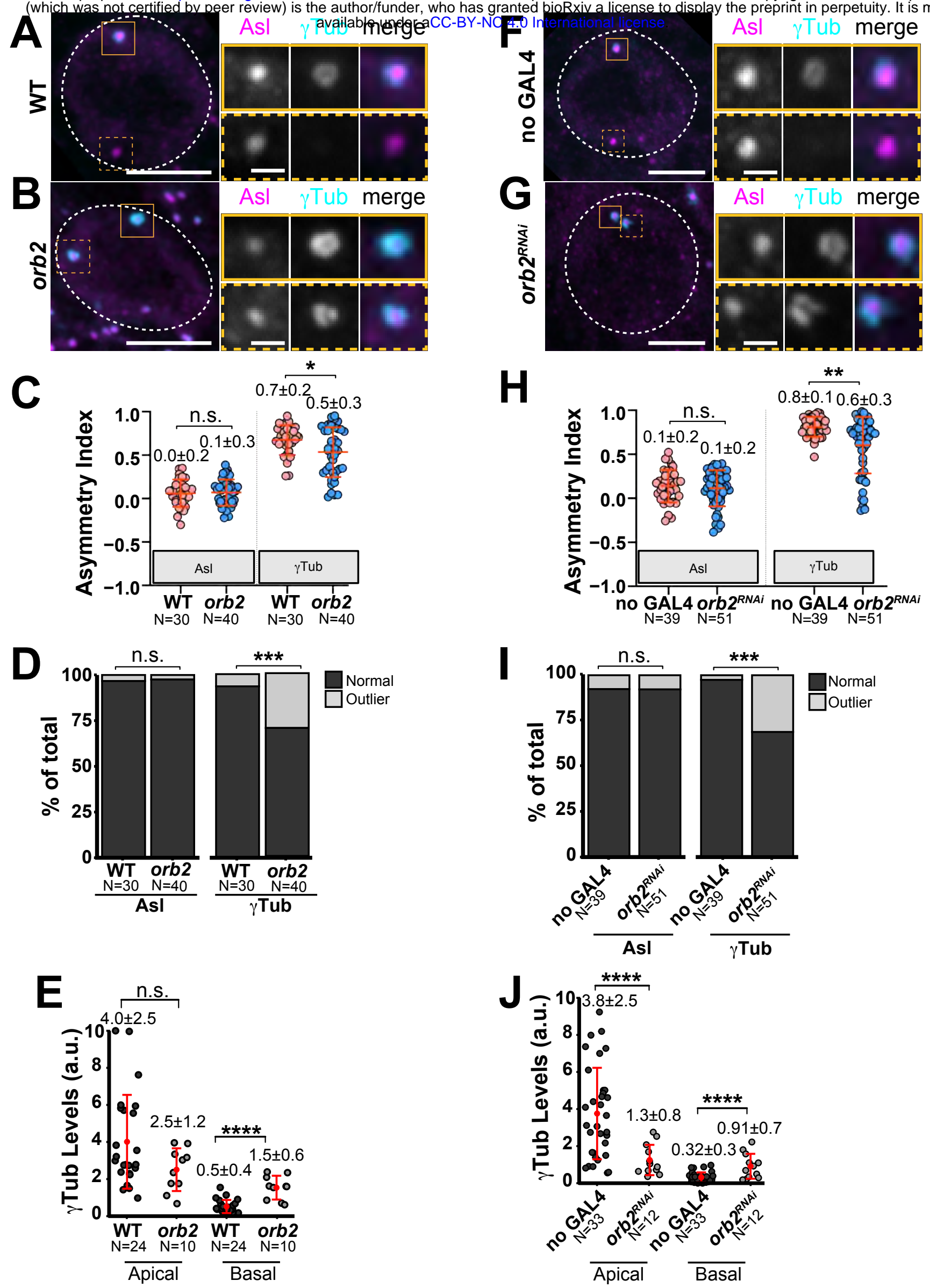

Robinson and Lerit Figure 1. orb2 is required for centrosome asymmetry 
bioRxiv preprint doi: https://doi.org/10.1101/2021.11.23.469707; this version posted November 23, 2021. The copyright holder for this preprint (which was not certified by peer review) is the author/funder, who has granted bioRxiv a license to display the preprint in perpetuity. It is made

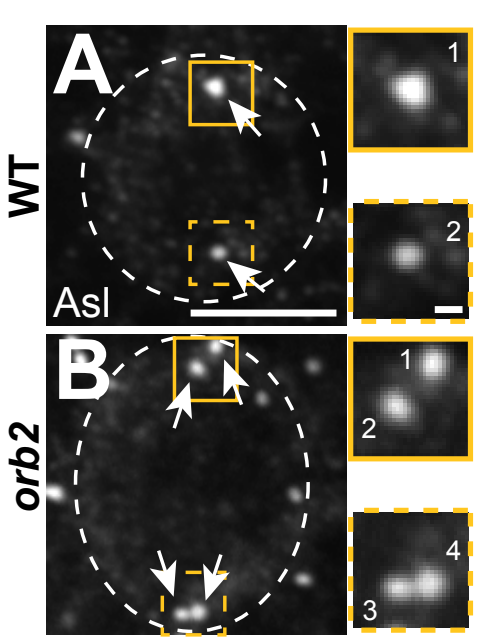

C $\square>2$ centrosomes

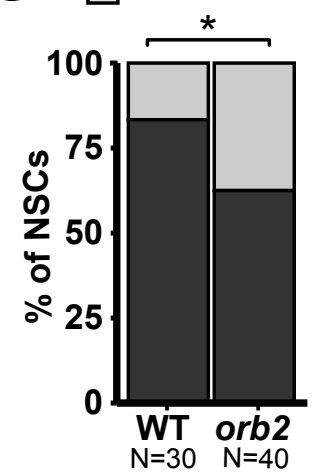

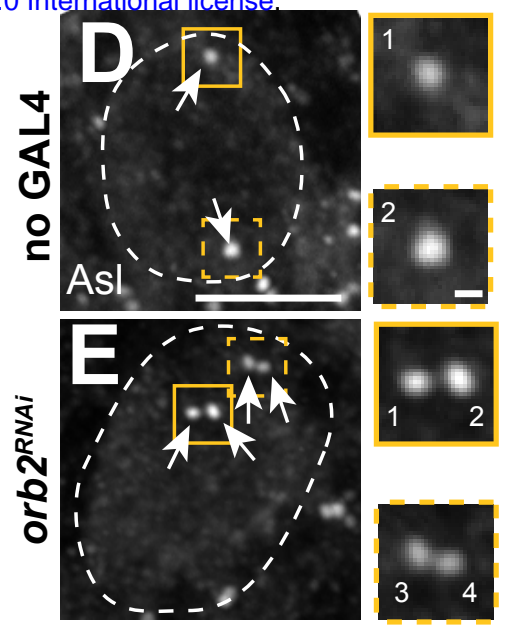

E $\square$ 2 centrosomes

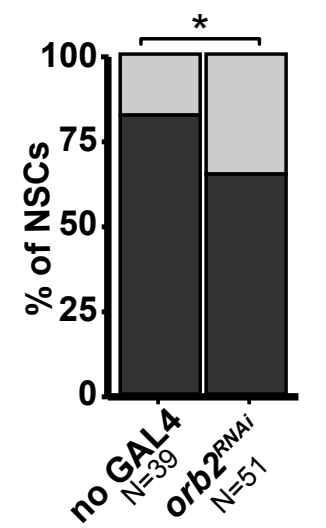

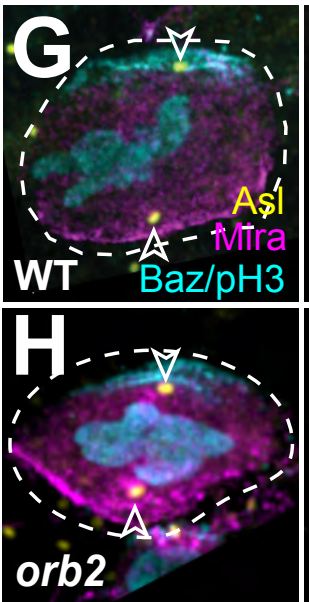

T
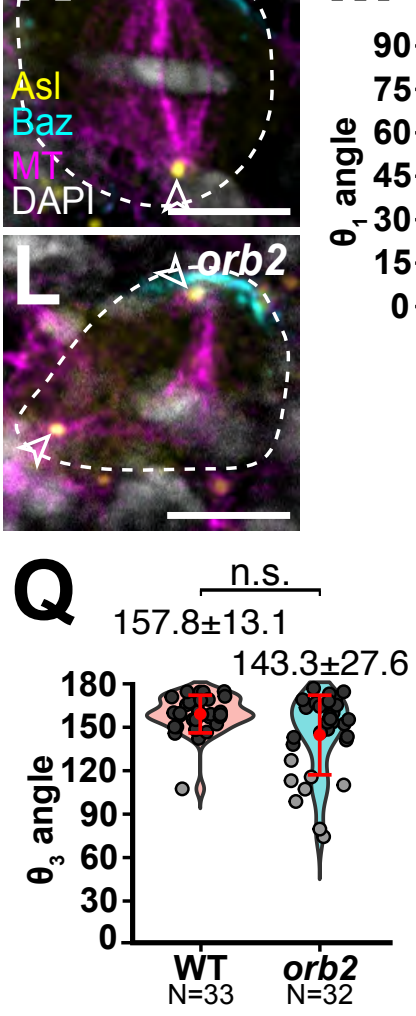
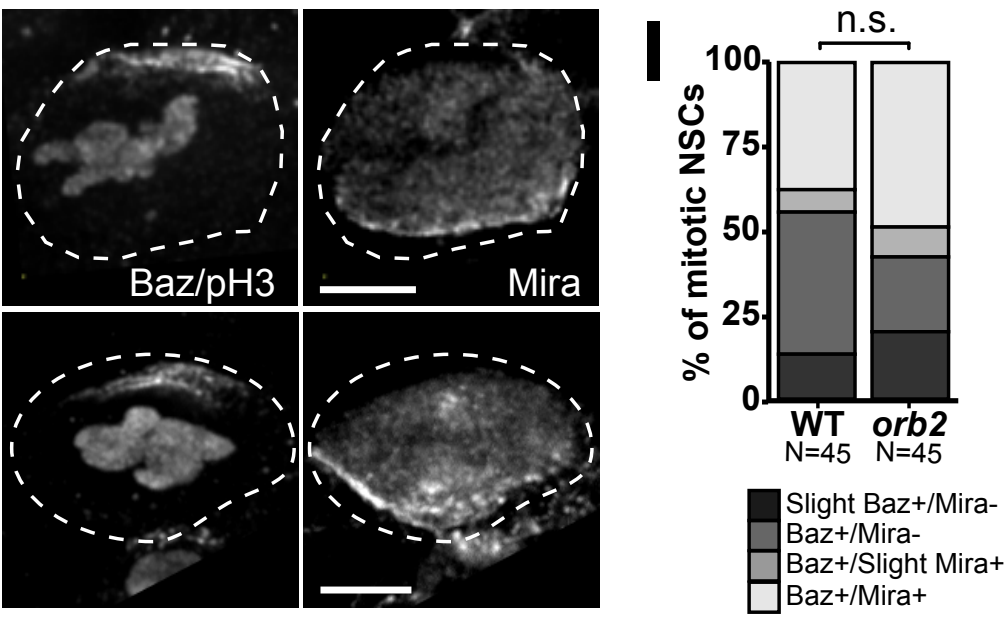

Baz+/Mira+
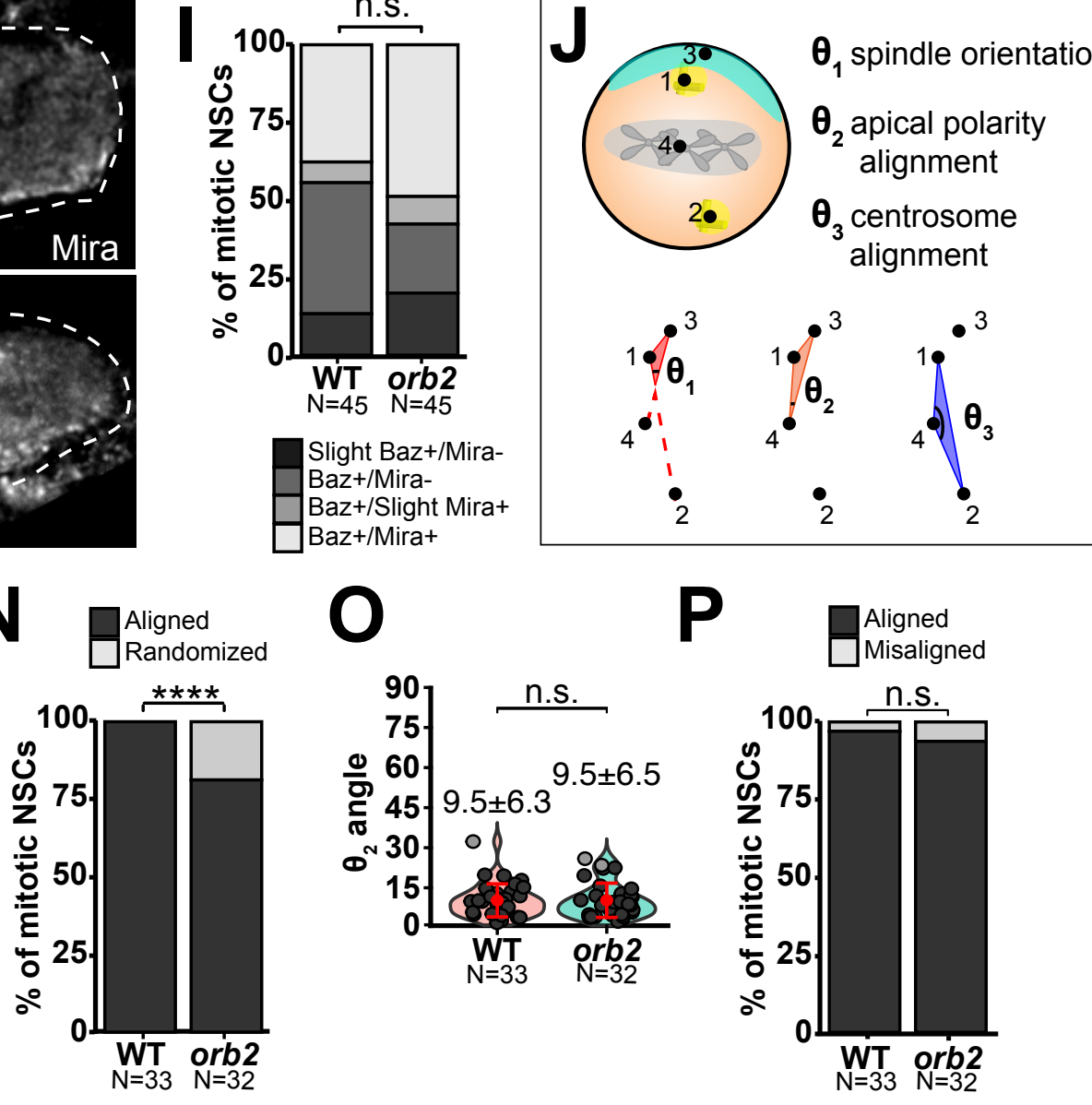

Robinson et al.

Figure 2. Orb2 is required for centrosome segregation and spindle morphogenesis

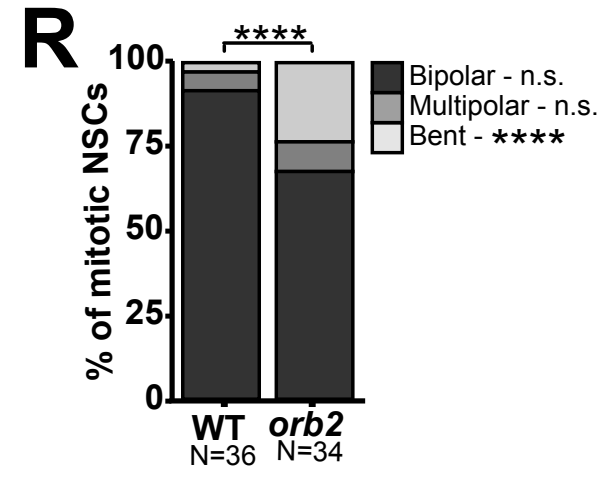


bioRxiv preprint doi: https://doi.org/10.1101/2021.11.23.469707; this version posted November 23, 2021. The copyright holder for this preprint (which was not certified by peer review) is the author/funder, who has granted bioRxiv a license to display the preprint in perpetuity. It is made available under aCC-BY-NC 4.0 International license.
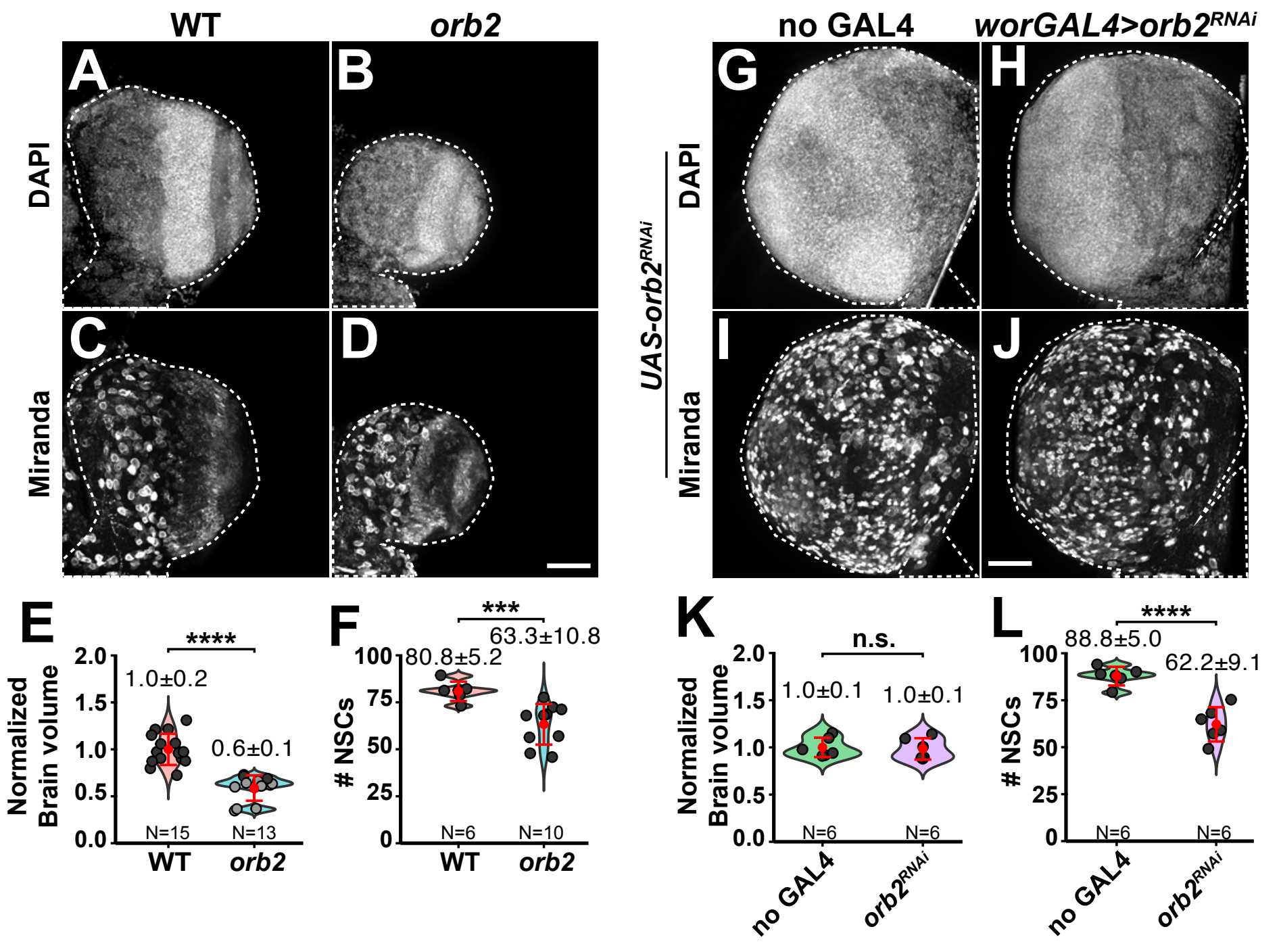

Robinson et al.

Figure 3. NSC-autonomous and non-autonomous Orb2 activities support neurodevelopment 


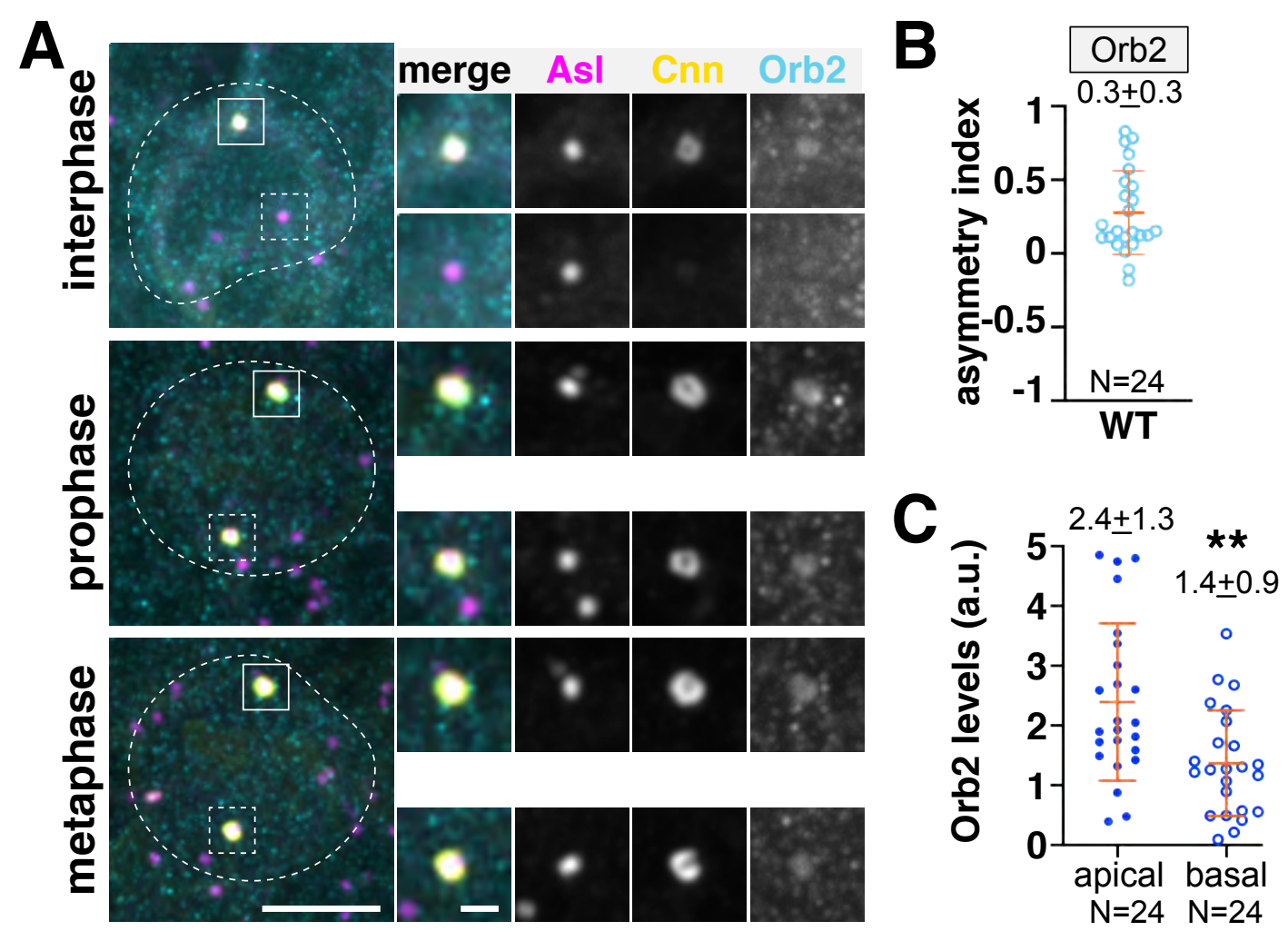

Robinson et al.

Figure 4. Orb2 localizes to active centrosomes 


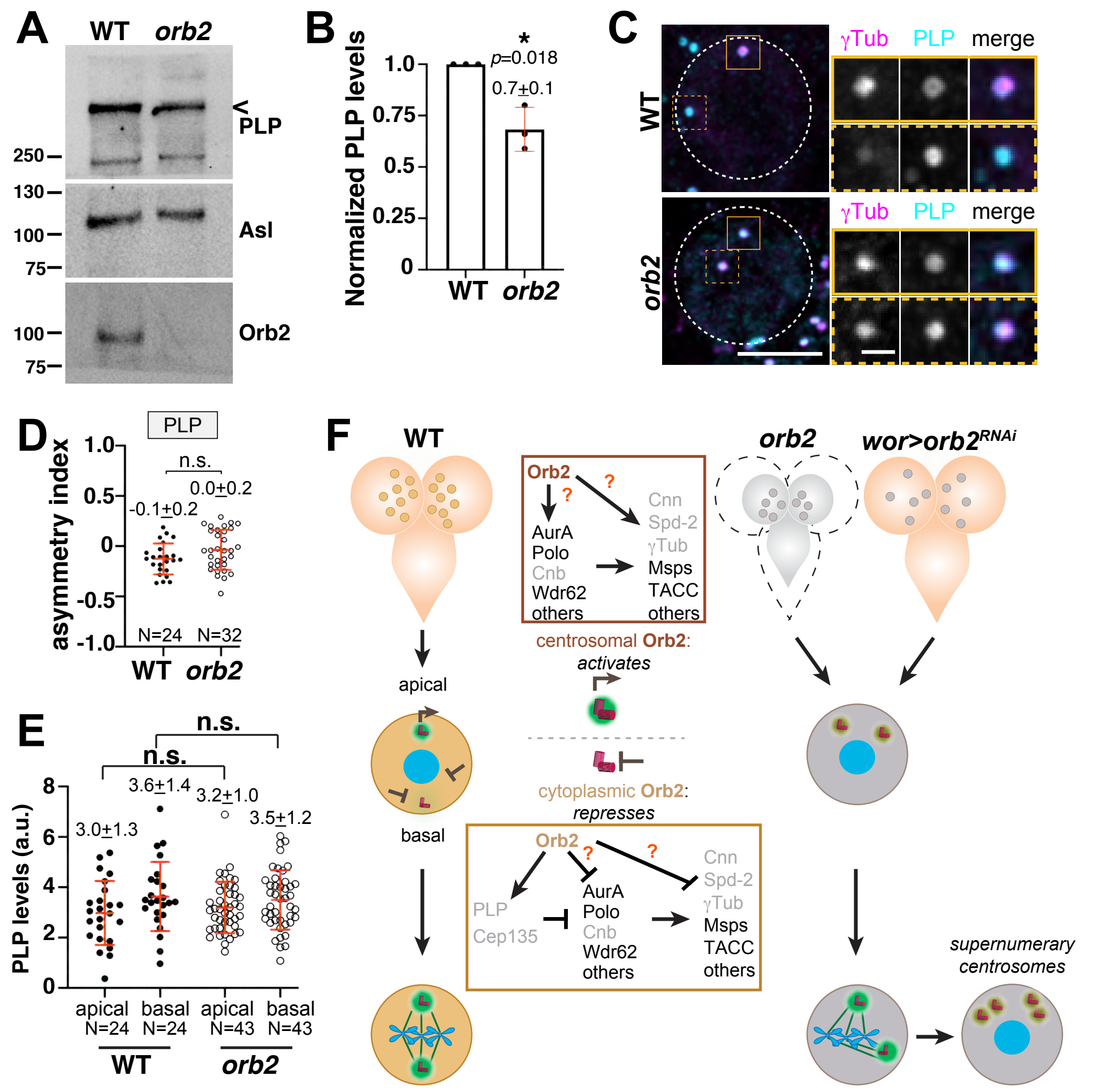



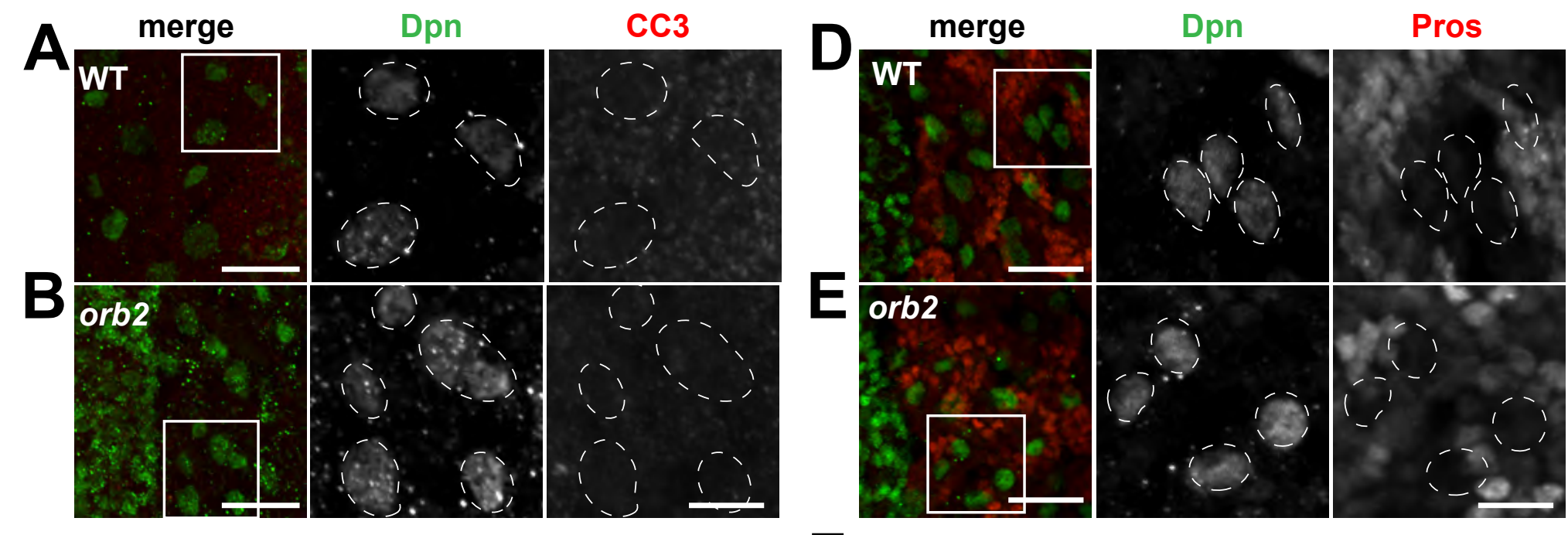

C
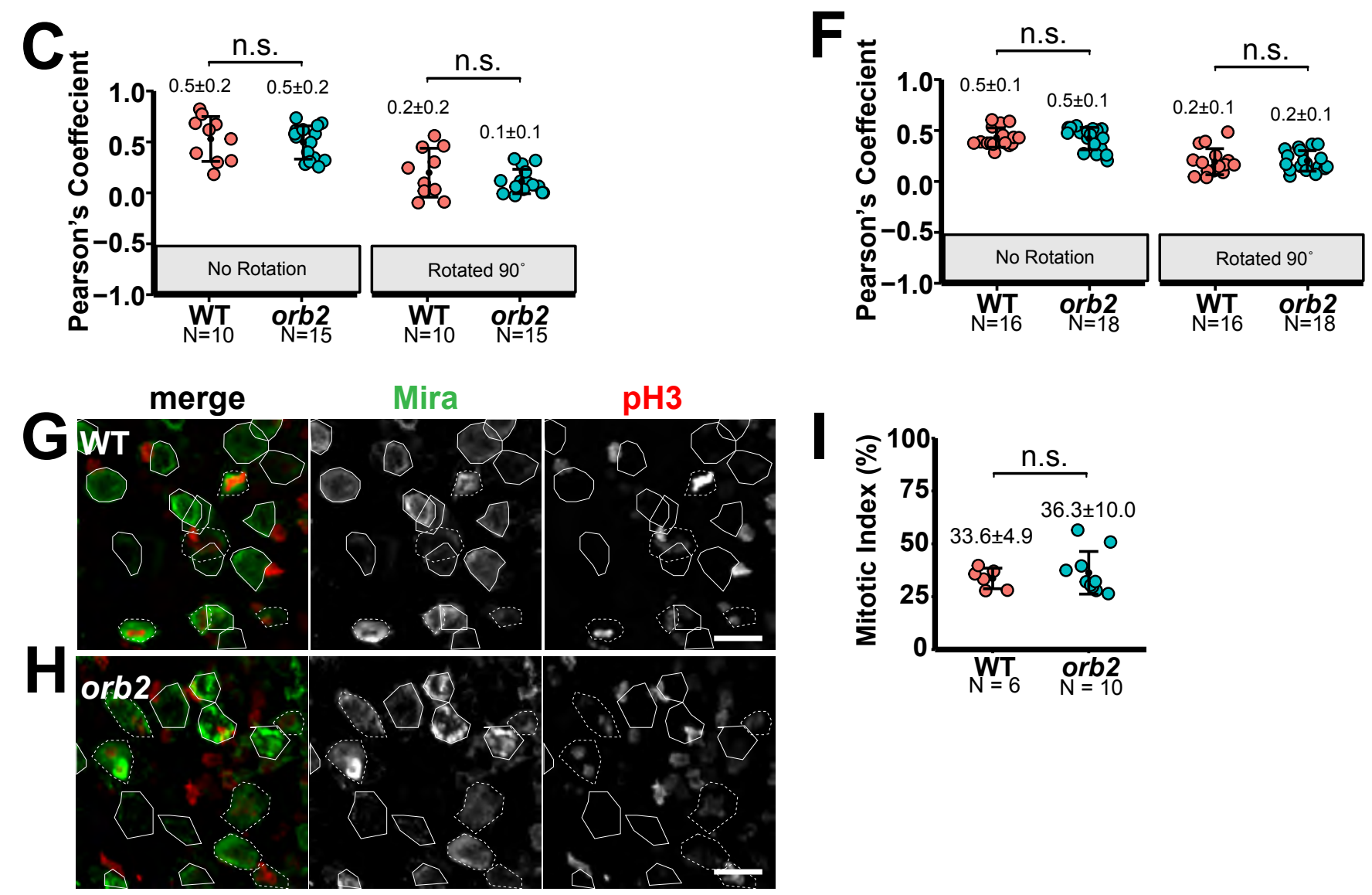

Robinson et al.

Figure S1. NSC differentiation and survival in WT vs. orb2 mutants 
bioRxiv preprint doi: https://doi.org/10.1101/2021.1123.469707; this version posted November 23, 2021. The copyright holder for this preprint (which was not certified by peer review) is the author/funder, who has granted bioRxiv a license to display the preprint in perpetuity. It is made available under aCC-BY-NC 4.0 International license.

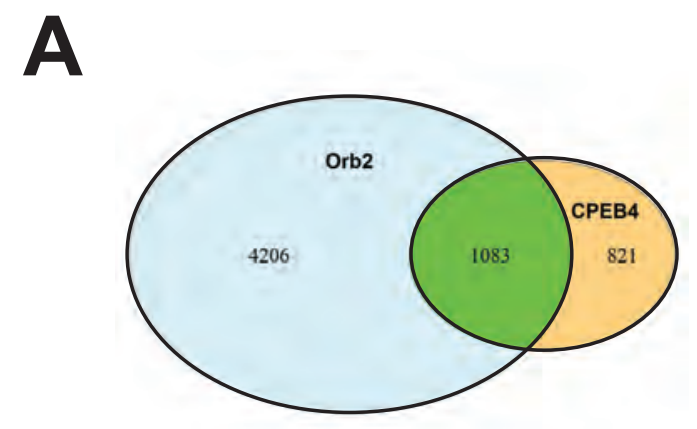

\section{B}

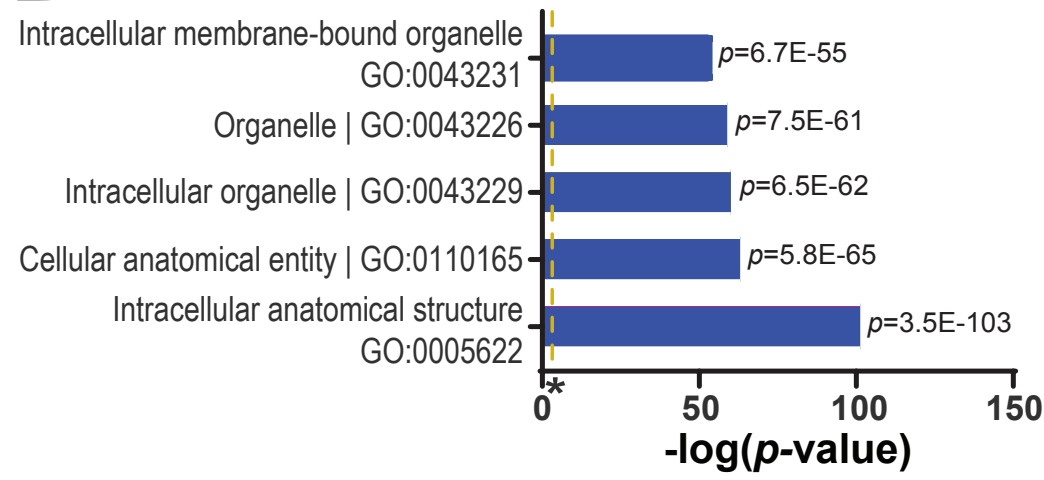

C

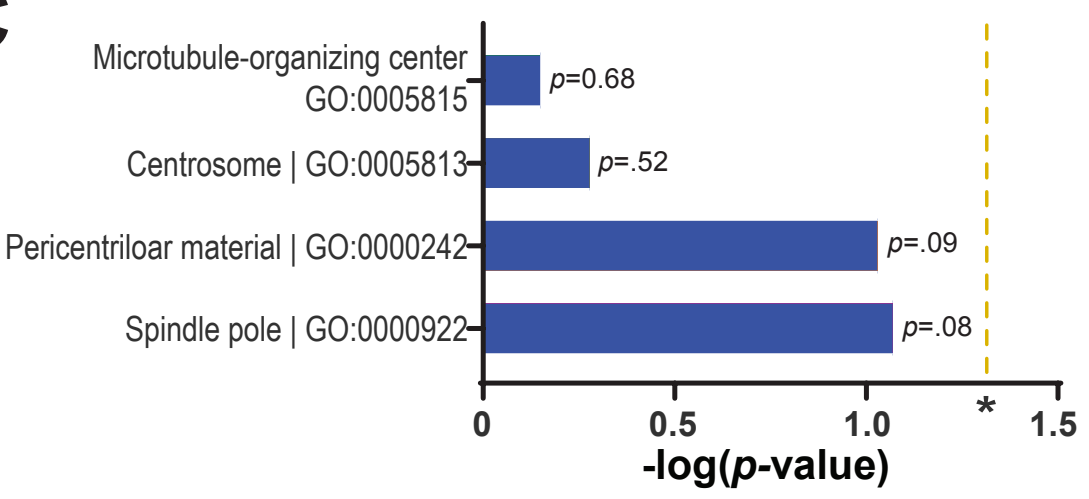

D

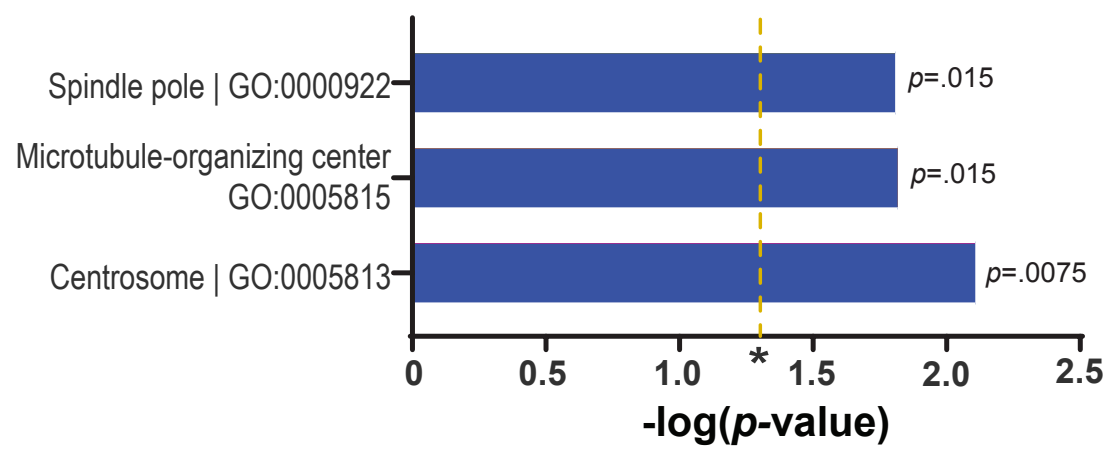

Robinson et al.

Figure S2. Ontological analysis of Orb2 and human CPEB4 